\title{
A Supra-galactic Conundrum: The Globular Clusters Colour Pattern in Virgo Galaxies
}

\author{
Juan C. Forte ${ }^{1,2 \star}$, Sergio A. Cellone ${ }^{2,3,6}$, María E. De Rossi ${ }^{7,8}$, Carlos Escudero ${ }^{2,3,4}$, \\ Favio R. Faifer ${ }^{2,3,4}$, Douglas Geisler ${ }^{5,9,10}$, Nélida M. González ${ }^{3}$, María C. Scalia ${ }^{3}$, \\ Leandro Sesto ${ }^{2,4}$, Analía V. Smith Castelli ${ }^{2,4}$, E. Irene Vega ${ }^{2,3}$ \\ ${ }^{1}$ Instituto Argentino de Matemática "Alberto P. Calderón", CONICET, Saavedra 15, 1083, CABA, Argentina \\ ${ }^{2}$ Consejo Nacional de Investigaciones Científicas y Técnicas, Godoy Cruz 2290, C1425FQB, CABA, Argentina \\ ${ }^{3}$ Facultad de Ciencias Astronómicas y Geofísicas, Universidad Nacional de La Plata, Paseo del Bosque, B1900FWA, La Plata, Argentina \\ ${ }^{4}$ Instituto de Astrofísica de La Plata (CCT-La Plata, CONICET-UNLP), Paseo del Bosque, B1900FWA, La Plata, Argentina \\ ${ }^{5}$ Departamento de Astronomía, Universidad de Concepción, Casilla 160-C, Concepción, Chile \\ ${ }^{6}$ Complejo Astronómico "El Leoncito" (CASLEO), CONICET-UNLP-UNC-UNSJ, San Juan, Argentina \\ ${ }^{7}$ Universidad de Buenos Aires, FCEN y Ciclo Básico Común, Buenos Aires, Argentina \\ ${ }^{8}$ CONICET-Universidad de Buenos Aires, Instituto de Astronomía y Física del Espacio, Buenos Aires, Argentina \\ ${ }^{9}$ Instituto de Investigación Multidisciplinario en Ciencia y Tecnología, Univ. de La Serena. Avenida Raúl Bitrán S/N, La Serena, Chile \\ ${ }^{10}$ Departamento de Física y Astronomía, Facultad de Ciencias, Univ. de La Serena. Av. Juan Cisternas 1200, La Serena, Chile
}

Accepted October 8, 2018.

\begin{abstract}
The presence of systematic modulations in the colour distributions in composite samples of globular clusters associated with galaxies in the Virgo and Fornax clusters has been reported in a previous work. In this paper we focus on the 27 brightest galaxies in Virgo, and in particular on $N G C$ 4486, the dominant system in terms of globular cluster population. The new analysis includes $\approx 7600$ cluster candidates brighter than $g=24.5$ (or $T_{1} \approx 23.70$ ). The results indicate the presence of the characteristic Virgo pattern in these galaxies and that this pattern is detectable over a galactocentric range from 3 to $30 \mathrm{Kpc}$ in $N G C$ 4486. This finding gives more support to the idea that the pattern has been the result of an external, still not identified phenomenon, capable of synchronizing the cluster formation in a kind of viral process, and on supra-galactic scales (also having, presumably, an impact on the overall star formation history in the entire Virgo cluster).
\end{abstract}

Key words: galaxies: star clusters: general

\section{INTRODUCTION}

The so called globular clusters (GCs) colour "bi-modality" has been a dominant paradigm for almost 25 years. In short, the term describes that in general, and with a few exceptions (see, for example, Sesto et al. 2016) the integrated colour distributions of $G C s$ in bright galaxies are dominated by two cluster populations: "blue" and "red" GCs (e.g. Brodie \& Strader 2006; Harris et al. 2017).

A first step towards exploring this issue beyond bimodality was presented in Forte (2017) (F2017 in what follows), who performed an analysis of composite $G C$ samples associated with galaxies in the Virgo and Fornax galaxy clusters. That work introduced an elementary pattern recogni-

* E-mail: planeta.jcf@gmail.com tion technique, based on the frequency of given colours in composite samples of $G C s$ in different galaxies.

This approach, using the $A C S$ photometry by Jordán et al. (2009) and Jordán et al. (2015), led to the discovery of distinct modulations in the $(g-z)_{o}$ (reddening corrected) $G C$ colours in both galaxy clusters, that were termed as the Template Virgo Pattern and Template Fornax Pattern ( $T V P$ and $T F P$, respectively). The Virgo pattern is characterized by the $(g-z)_{o}$ colours listed in Table 1 of F2017 (namely, 0.74, 0.85, 0.95, 1.05, 1.13, 1.21, 1.29, 1.39, 1.48, 1.60 and 1.72). Values in brackets along the following text identify a given colour in the $T V P$.

The basic idea is that composite $G C$ samples may enhance the presence of possible common features as, for example, "peaks" or "valleys" in the GCs colour distribution (GCCD in what follows). The colour modulations are most evident for $G C s$ in moderately bright early type galaxies, with $M_{g}$ from -20.2 to -19.2 , but they were also found in 
some $G C$ sub - samples of the brightest galaxies in these clusters (e.g. NGC 4474 and NGC 4486 in Virgo; NGC 1399 and NGC 1404 in Fornax). The TVP is in fact a "die-hard" feature as it survives after splitting the $G C s$ sample in terms of cluster brightness, galaxy brightness or spatial position as described in F2017.

After rejecting the incidence of field contamination, instrumental, and statistical effects, the conclusion in F2017 was that the colour patterns are not spurious but have a physical entity. On statistical grounds, Monte Carlo models along the lines described in Forte, Faifer \& Geisler (2007) ( $F F G 07$ in what follows), indicate that the probability of having a colour pattern with the properties of the $T V P$ as the result of statistical fluctuations in a $G C$ sample with $\approx$ 1500 objects, is practically null (i.e., below 0.001).

This paper focusses on GCs in the brightest Virgo cluster galaxies and extends the previous uni-dimensional analysis (colour) to a bi-dimensional space (colour-magnitude). In particular, we study the 27 brightest galaxies in Virgo ( $M_{g}$ from -23.0 to -19.2) since, for fainter galaxies, the $G C s$ colour pattern is not evident and appears only marginally in some cases.

The galaxy sample now includes 12 giant galaxies (brighter than $M_{g}=-20.2$ ), not discussed in F2017, and among them, $N G C 4486$, the dominant system in terms of the $G C$ population. The outer regions of this system are explored using previously published Washington photometry (FFG07).

\section{REVISITING THE TEMPLATE VIRGO PATTERN.}

In this section we revisit the so called Template Virgo Pattern using the same pattern recognition technique presented in F2017 although introducing two changes in the strategy of the data analysis.

First, by performing an analysis of the $H S T-A C S$ photometric errors given in Jordán et al. (2009), we found that the colour pattern can be more clearly detected adopting a limiting magnitude $g_{o}=24.5$, i.e., half a magnitude brighter than the cut-off in F2017. With this magnitude limit, the "colour spread function" (CSF; the function that results after combining photometric errors and the smoothing gaussian kernel), allows the resolution of colour peaks at a level of $\approx 0.07$ mags. in $(g-z)_{o}$.

Second, the finding routine was run on each of the 27 brightest galaxies in Virgo, instead of grouping galaxies within a sampling window defined in the colour vs. absolute magnitude diagram. This approach eventually removes the chance that the observed pattern is a result of the type of adopted $G C$ sampling.

The galaxy sample is listed in Table 1 and is ordered by decreasing brightness, as in Table 1 of Ferrarese et al. (2006), who present structural photometric parameters for these galaxies. Table 1 gives the galaxy identification, $(g-z)$ o colour shifts (see below), galactocentric range, and number of sampled GCs with $g_{o}=20.0$ to 24.5 .

We remark that the $G C$ sample in Table 1 is a factor of three times larger than that defined by clusters in the moderately bright galaxies $\left(M_{g}\right.$ from -20.2 to -19.2$)$ discussed in F2017.

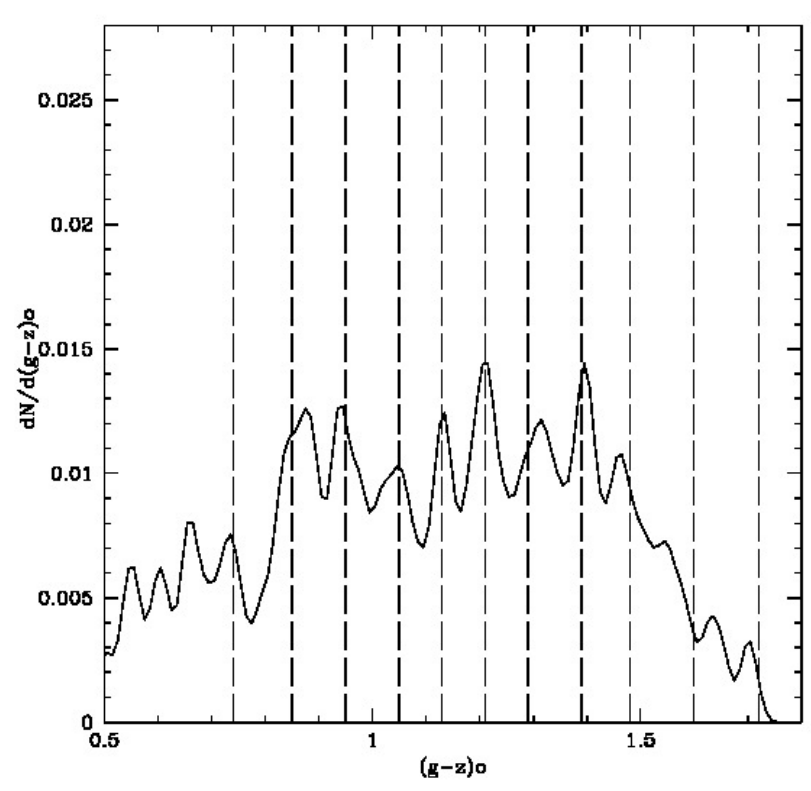

Figure 1. Smoothed relative $(g-z) o$ colour frequency corresponding to 311 peaks found on the $G C$ sample belonging to the 27 galaxies listed in Table 1 . The cluster colours are the original photometric data (corrected by interstellar extinction). Vertical lines indicate to the $T V P$ colours.

Running the colour peak finding routine (PFR in what follows) on each individual galaxy shows that, as a general trend in giant galaxies, the colour patterns are difficult to find within galactocentric radii $\left(R_{\text {gal }}\right)$ smaller than $40^{\prime \prime}$. For these galaxies we set a galactocentric search range from $40^{\prime \prime}$ to $100^{\prime \prime}$. For the remaining ones, the galactocentric domain was set from $0^{\prime \prime}$ to $100^{\prime \prime}$.

The results from the PFR reveals the presence of 311 colour peaks (corresponding to a total of 4289 GCs) whose smoothed distribution, adopting a Gaussian kernel of 0.015 mag. (as in F2017), is shown in Fig. 1. This diagram, normalized by the total number of colour peaks, indicates the relative frequency of each colour peak (irrespective of the number of GCs with that colour) and shows nine coincidences with the $T V P$. The differences between each colour peak and that of the nearest colour in the $T V P$, leads to a $r m s=0.016$. The situation is poorly defined regarding the reddest $T V P$ features, at [1.60] and [1.72].

The nature of the peaks outside the $G C s$ colour range is difficult to asses. In an speculative way, these features could arise in field objects as they do not show any obvious spatial concentration towards the galaxy centres.

The outputs from the $P F R$ also indicate that, in some cases, the whole $T V P$ is detectable although with some small shifts in colour. $N G C 4472$, the brightest Virgo galaxy, is a good example as its $G C C D$ shows all the $T V P$ features, although shifted by +0.05 mag in $(g-z) o$. Similar systematic shifts are also detectable in other galaxies with well populated GCs systems.

The shifts that bring a given pattern into agreement with the TVP (by minimizing the $r m s$ of the differences between each colour peak and the nearest one in the $T V P$ ), are listed in Table 1.

As noted in F2017, these shifts are within the uncer- 


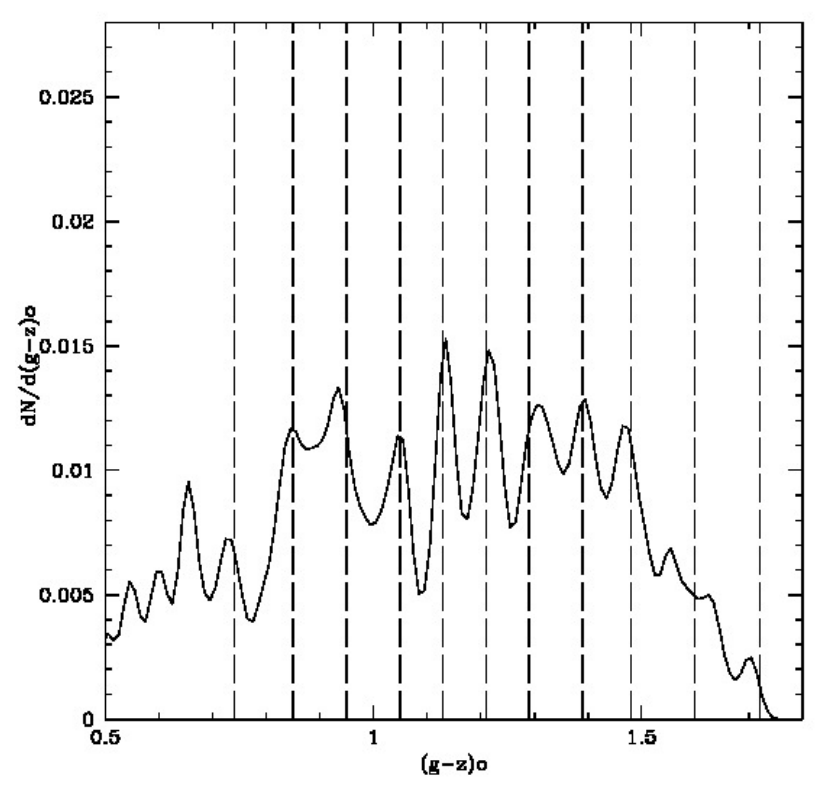

Figure 2. Smoothed relative $(g-z) o$ colour frequency corresponding to 311 peaks found on the $G C$ sample belonging to the 27 galaxies listed in Table 1. Some of the cluster colours (corrected by interstellar extinction) have been shifted according to the values listed in that table. Vertical lines indicate the TVP colours.

tainties in the adopted interstellar reddening. In fact, the $r m s$ of the original infra-red emission vs. colour excess map calibration by Schlafly \& Finkbeiner (2011) is close to \pm 0.03 mag. in $E(B-V)$ that corresponds to \pm 0.05 mag. in $(g-z)$.

The results from the PFR, after adopting the colour shifts listed in Table 1, lead to the relative frequency distribution displayed in Fig. 2. This diagram, compared to the previous one, shows an overall increase of the contrast of the peaks and also a decrease of the rms value to 0.010 .

\section{THE COMPOSITE GLOBULAR CLUSTERS COLOUR DISTRIBUTIONS IN THE 27 BRIGHTEST VIRGO GALAXIES.}

In what follows we present the GCCDs (normalized by total numbers of $G C s$ ), both in the discrete and smoothed histogram versions. The colour-bin size (0.04 mag) and the Gaussian kernel (0.015 mag) are those discussed in F2017.

The differences between the colour peaks found by the pattern recognition routine and those of the nearest colour in the $T V P$ definition, leads to a $r m s$ value that is an indicator of the agreement between both patterns. This $r m s$ is calculated for colour peaks within the colour range $(g-z)$ o from 0.80 to 1.60 , characteristic of old $G C s$.

In order to derive the composite $G C C D s$, the clusters belonging to the galaxies listed in Table 1 were combined in three different groups (separated by blank lines in the table) containing approximately the same number of $G C s$ each.

The GCCD corresponding to galaxies brighter than $M_{g}=-21.8$, including $N G C 4472,4486$ and 4649 (with 1447 $G C s)$, is displayed in Fig. 3. The vertical lines in this figure

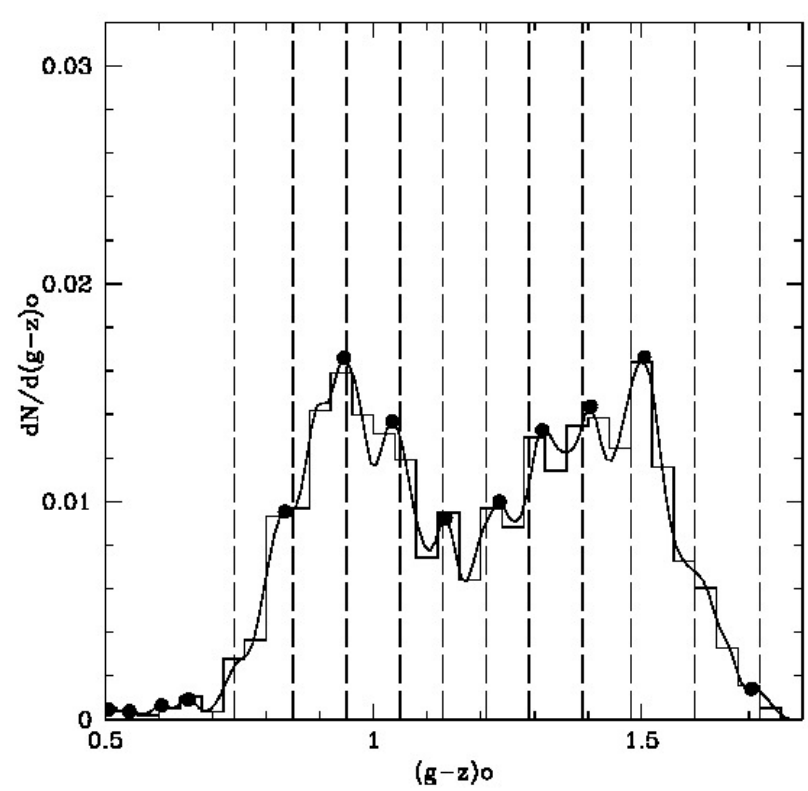

Figure 3. $(g-z) o$ colour distribution corresponding to a composite $G C$ sample that includes 1447 objects in the three brightest Virgo giants: $N G C 4472,4486$ and 4649 and within a galactocentric range from $40^{\prime \prime}$ to $100^{\prime \prime}$. The $G C$ colours have been shifted according to Table 1. Vertical lines correspond to the TVP colours. Dots indicate the colour peaks found by the $P F R$.

(and in following ones) correspond to the $T V P$ colours, while dots indicate the colour peaks found by the PFR. The rms corresponding to the differences between the detected peaks and those that define the $T V P$ is \pm 0.016 (eight peaks).

In this case the feature at [0.85] is barely detectable. This is consistent with results available in the literature (see, for example, Escudero et al. 2018) showing that the inner regions of giant galaxies are usually dominated by red $G C s$ while the bluer globulars are less abundant.

Fig. 4 is the $G C C D$ corresponding to nine less massive giants $\left(M_{g}=-20.2\right.$ to -21.8 ; from $N G C 4406$ to 4762 in Table 1; 1411 GCs). This group has a slightly larger rms $( \pm 0.018 \mathrm{mag})$ compared with the previous one $( \pm 0.016)$, and again, the colour peak at [0.85] is not evident.

As expected, the reddest $T V P$ peaks in this galaxy group are less prominent than those in the more massive, and more chemically enriched, ones displayed in Fig. 3.

Finally, the GCCD corresponding to 1431 GCs in fifteen moderately bright galaxies $\left(M_{g}=-19.2\right.$ to -20.2$)$ is depicted in Fig. 5. This figure shows all the TVP peaks from [0.85] to [1.39] and yields an rms of only $0.007 \mathrm{mag}$. This group shows a prominent blue peak at [0.85]. This can be expected since, being smaller in angular size, their outer regions fall within the real coverage of the ACS photometry.

The determination of the TVP colours in F2017 was based on a galaxy sample that included galaxies fainter than $\mathrm{Mg}=-20.2$. However, this section shows that this pattern can be also consistently recognized in the composite $G C C D$ corresponding to the twelve giant galaxies listed in Table 1. Furthermore, the two brightest galaxies in Virgo (NGC4472, and 4486) have individual $G C C D s$ where the $T V P$ pattern is easily recognizable (see below). 


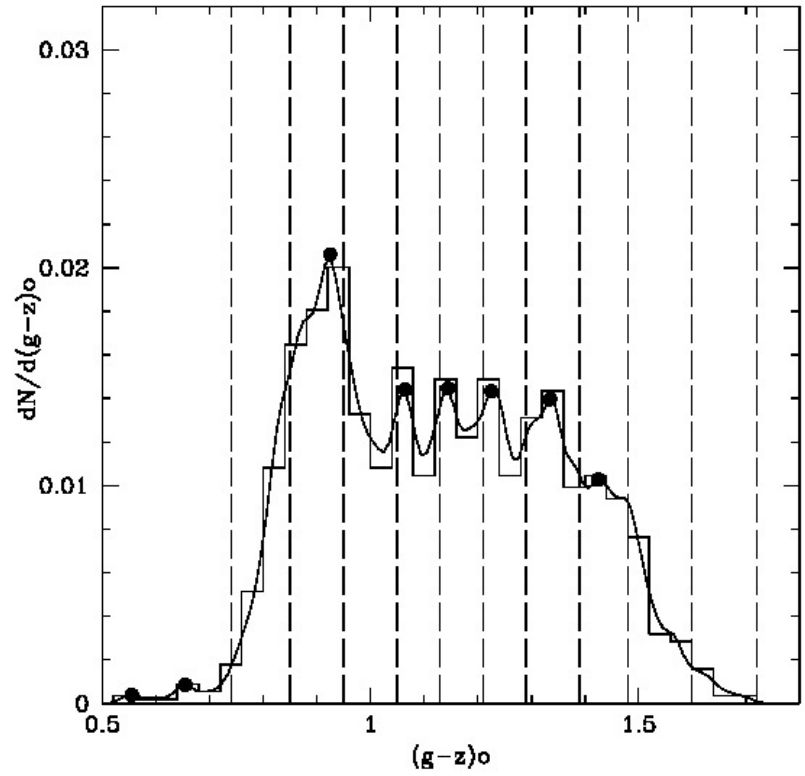

Figure 4. $(g-z) o$ colour distribution corresponding to a composite sample of 1411 GCs belonging to nine giant galaxies in Table 1 (from NGC 4406 to 4762). Vertical lines correspond to the TVP colours. Dots indicate the colour peaks found by the PFR.

The presence or absence of some colour peaks, at the blue or red edges of the GCCDs, can be explained in terms of the different chemical enrichment levels that characterize each galaxy group. In general, the most massive galaxies (with high chemical enrichment) will show red TVP components that become less evident or absent in lower mass galaxies.

Conversely, low mass galaxies will exhibit blue $T V P$ components (that are less prominent in the inner regions of massive galaxies). The smoothed colour magnitude diagram for the 4289 GCs sample corresponding to all the galaxies listed in Table 1, is shown in Fig. 6.

For constructing this diagram, the $g_{o}$ magnitudes and $(g-z)_{o}$ colours were mapped as components of a $501 \times 501$ matrix defined between $g_{o}=20.0$ and 25.0, and $(g-z)_{o}$ from 0.5 to 2.0. The IRAF routine gauss was run on this matrix, generating a FITS image after adopting a colour kernel of $0.015 \mathrm{mag}$, and a $g$ magnitude kernel of $0.25 \mathrm{mag}$. The first value is that used in the F2017 uni-dimensional analysis while the second one was set after trial - and-error runs (with $g_{o}$ magnitude kernels ranging from 0.10 to $0.50 \mathrm{mag}$ ) aiming at enhancing the presence of the colour pattern.

Fig. 6 is a false-colour display of the FITS image (after adopting a proper minimum/maximum range and contrast) and shows a highly structured $G C C D$. The contour of the Gaussian kernel is shown at the upper right in this diagram. The expectation is that, if the colour patterns are not random effects, they should show some coherence in the colour magnitude plane. In fact, Fig. 6 indicates that the colour modulations are not the result of localized data "clumps" but, rather, distinct structures spanning different ranges in apparent magnitude and coincident with the TVP colours, shown as vertical lines (see Section 6). The "widths" of these

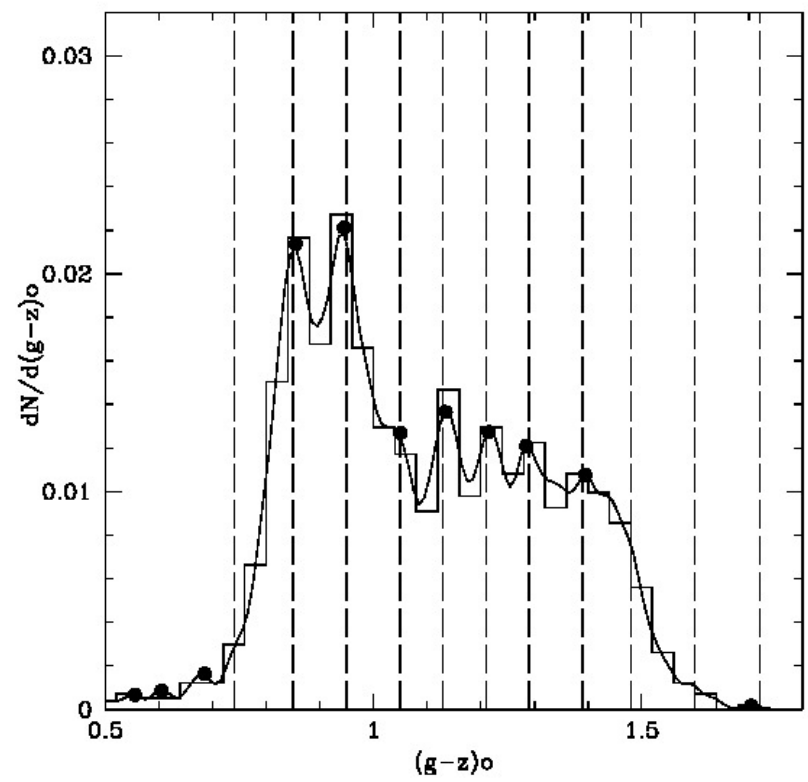

Figure 5. $(g-z) o$ colour distribution for 1431 GCs corresponding to fifteen moderately bright galaxies listed in Table 1 (from NGC 4459 to 4578$)$. Vertical lines correspond to the TVP colours. Dots indicate the colour peaks found by the PFR.

structures are in fact set by a combination of the $C S F$ and the convolving kernel.

A remarkable feature in the last diagram is the presence of two blue peaks $[0.85 ; 0.95]$ that appear in most of the individual galaxies listed in Table 1.

\section{THE TEMPLATE VIRGO PATTERN IN NGC 4486}

$N G C 4486$ is a well known paradigm of the so called "high $S_{n}$ " galaxies (e.g. Tamura et al. 2006) and is the dominant system in Virgo in terms of its GCs population. F2017 noted that the $T V P$ can be detected at some galactocentric radii and position angles ranges (see fig. 22 and 23 in that paper).

The presence of the $T V P$ is seen in Fig. 7 where the $G C C D$ corresponds to 762 clusters with $A C S$ photometry and galactocentric radii from $40^{\prime \prime}$ to $90^{\prime \prime}$. This diagram shows seven coincidences with the TVP with an rms of 0.019 . A disagreement is seen for the bluest GCs for which the [0.85] feature appears some 0.04 mag. redder than expected. The behaviour of the bluest $G C$ colours is similar to that described in the previous discussion regarding blue $G C s$ in the inner regions of massive galaxies.

In what follows we discuss the eventual detectability of the TVP in the outer regions of NGC 4486 using the photometry presented by FFG07. These ground based observations were obtained with the the Mayall $4-m$ telescope at $K P N O$, and adopting the $\left(C-T_{1}\right)$ colour index defined with these two passbands of the Washington photometric system.

A key issue in this context is a reliable determination of the $(g-z) o$ vs $\left(C-T_{1}\right)_{o}$ colour-colour relation, aiming at obtaining the equivalent $T V P$ colours in the $\left(C-T_{1}\right)_{o}$ colour scale. This can be carried out using the Gemini-griz 


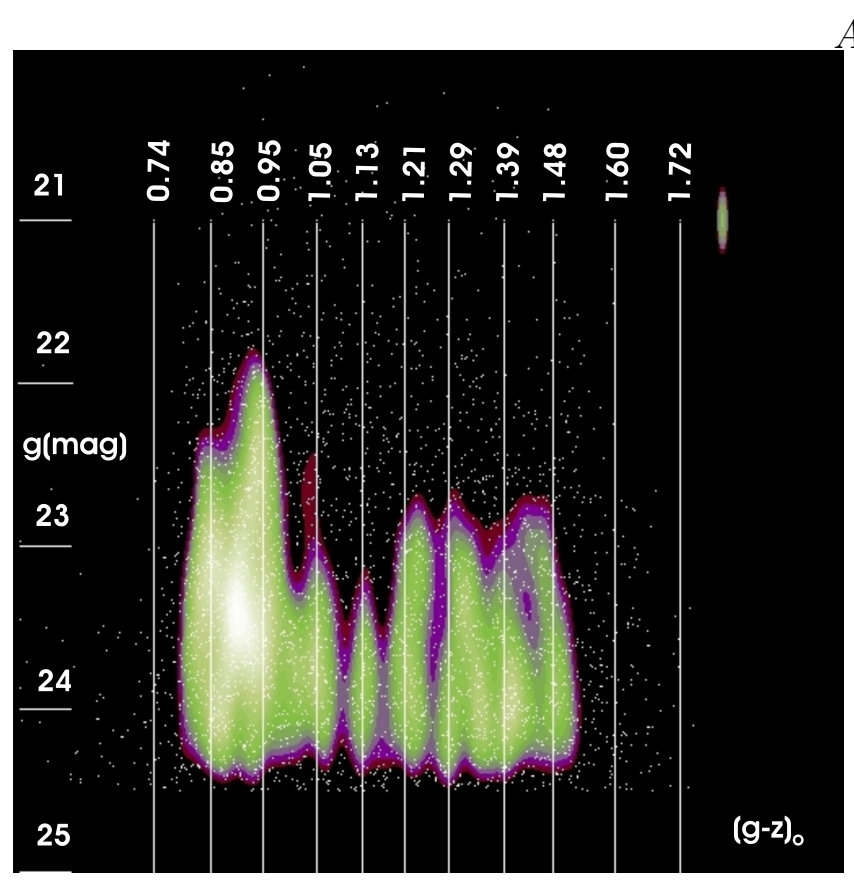

Figure 6. Smoothed colour-magnitude diagram for 4289 GCs corresponding to all the galaxies listed in Table 1 (within the indicated galactocentric ranges, and adopting the indicated colour shifts). The vertical lines correspond to the TVP colours. White dots represent individual GCs. The contour of the convolving kernel is shown at upper right.

Table 1. GCs samples ( $g_{o}=20.0$ to 24.5$)$ in the 27 brightest Virgo galaxies.

\begin{tabular}{|c|c|c|c|}
\hline NGC & $(g-z)_{o}$ shift & Gal. range " & $\mathrm{N}$ of $\mathrm{GCs}$ \\
\hline 4472 & -0.05 & $40-100$ & 330 \\
\hline 4486 & -0.00 & $40-100$ & 762 \\
\hline 4649 & -0.02 & $40-100$ & 355 \\
\hline 4406 & +0.05 & $40-100$ & 172 \\
\hline 4382 & +0.03 & $40-100$ & 176 \\
\hline 4374 & -0.02 & $40-100$ & 203 \\
\hline 4365 & 0.00 & $40-100$ & 304 \\
\hline 4526 & +0.02 & $40-100$ & 97 \\
\hline 4621 & +0.01 & $40-100$ & 144 \\
\hline 4552 & -0.02 & $40-100$ & 176 \\
\hline 4473 & 0.00 & $40-100$ & 99 \\
\hline 4762 & 0.00 & $40-100$ & 40 \\
\hline 4459 & 0.00 & $0-100$ & 128 \\
\hline 4442 & 0.00 & $0-100$ & 128 \\
\hline 4754 & 0.00 & $0-100$ & 61 \\
\hline 4267 & 0.00 & $0-100$ & 129 \\
\hline 4371 & 0.00 & $0-100$ & 120 \\
\hline 4570 & 0.00 & $0-100$ & 90 \\
\hline 4435 & 0.00 & $0-100$ & 120 \\
\hline 4660 & 0.00 & $0-100$ & 143 \\
\hline 4530 & 0.00 & $0-100$ & 113 \\
\hline 4564 & 0.00 & $0-100$ & 113 \\
\hline 4340 & 0.00 & $0-100$ & 29 \\
\hline 4417 & 0.00 & $0-100$ & 61 \\
\hline 4638 & 0.00 & $0-100$ & 58 \\
\hline 4478 & 0.00 & $0-100$ & 90 \\
\hline 4578 & 0.00 & $0-100$ & 48 \\
\hline
\end{tabular}

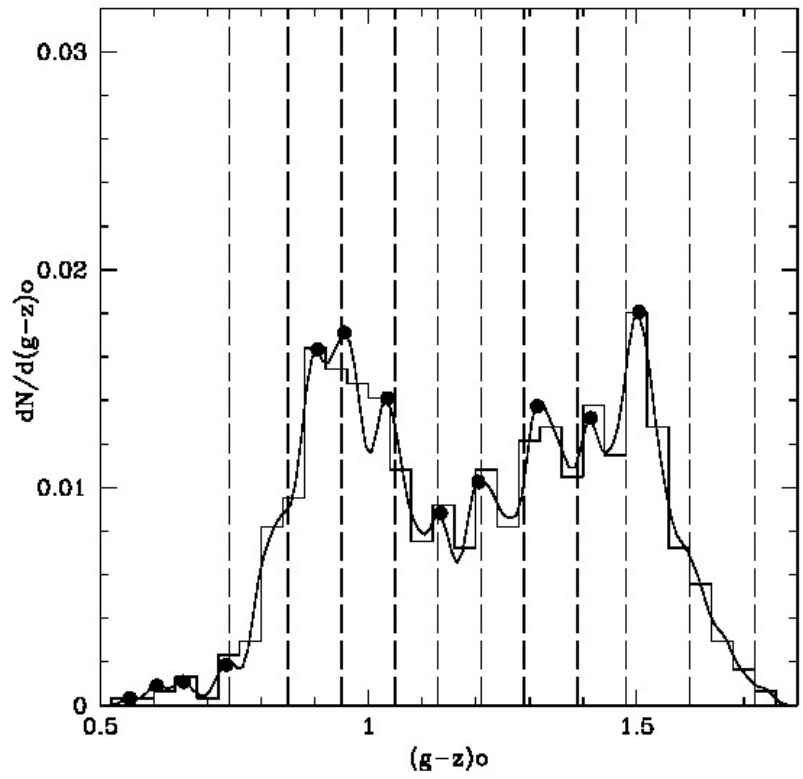

Figure 7. Discrete bin and smoothed $(g-z) o$ colour distribution for $762 G C s$ with $A C S$ photometry and galactocentric radii from $40^{\prime \prime}$ to $90^{\prime \prime}$ in NGC 4486. Vertical lines correspond to the TVP colours. Black dots indicate the colour peaks found by the $P F R$.

photometry presented in Forte et al. (2013) for an offset field in $N G C$ 4486. This field includes $522 G C$ candidates that also have Washington $C$ and $T_{1}$ photometry given in FFG07.

As a first step, an analysis of the griz colours was performed in an attempt to remove field interlopers. This procedure left 457 objects with colours that are fully compatible with those of old $G C s$. The $(g-z) o$ vs $\left(C-T_{1}\right)_{o}$ colourcolour diagram for these objects is shown in Fig. 8. The straight line in this figure corresponds to a bi-sector fit (i.e., taking into account the photometric errors on both axes):

$\left(C-T_{1}\right)_{o}=1.287 \cdot(g-z)_{o}+0.027$

(with coefficient uncertainties of \pm 0.012 and \pm 0.014 , respectively).

The analysis of the residuals of this fit does not show any systematic trend or deviation from linearity with colour.

This relation then allows a link between the $(g-z)_{o}$ $T V P$ colours and those in the $\left(C-T_{1}\right)_{o}$ colour scale, with uncertainties below \pm 0.015 mag., yielding: $0.98,1.12,1.25$, $1.38,1.48,1.58,1.69,1.82,1.93,2.09,2.23$.

\section{THE GCCD IN THE OUTER REGIONS OF NGC 4486}

\subsection{The GCCD in NGC 4486 for galactocentric radii between 90 and 240 arcsec.}

The $\left(C-T_{1}\right)_{o} G C C D$ for 2048 cluster candidates with galactocentric radii from $90^{\prime \prime}$ to $240^{\prime \prime}$ (with a complete areal coverage) is displayed in Fig. 9. This sample corresponds to a magnitude range $T_{1 o}$ from 20.0 to 24.0 , i.e., some 0.5 mag. brighter than the photometric cut-off in FFG07. The PFR detects seven peaks compatible with the $T V P$ [in the colour 


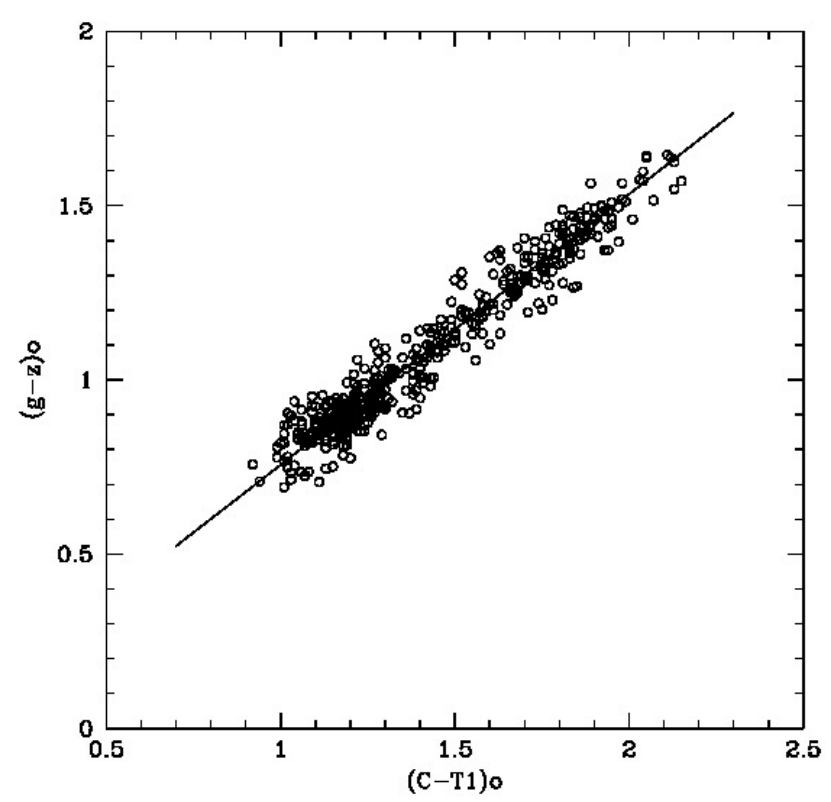

Figure 8. The $(g-z) o$ vs. $\left(C-T_{1}\right)_{o}$ colour relation. Dots correspond to $457 G C$ candidates, taken from Forte et al. (2013), whose colours are compatible with those of old GCs (see text).

range from $\left(C-T_{1}\right)_{o}=1.12$ to 1.82$]$ with an $r m s$ of 0.023 mag. (or $0.018 \mathrm{mag}$. in a $(g-z)$ colour scale). This value is comparable with those that characterize the $G C C D s$ in Virgo galaxies, as discussed in Section 2 and 3. In fact, there is a clear similitude between Fig. 9 and Fig. 5, corresponding to $G C s$ associated with the moderately bright galaxies. This similitude indicates that, as a whole, the NGC4486 $G C s$ in this galactocentric range, have chemical abundances comparable to those in the bright galaxies sample listed in Table 1.

Fig. 9 also shows a feature at $\left(C-T_{1}\right)_{o}=1.04$ that has not been found in the other galaxies and whose nature is discussed in Section 6.

Colour peaks redder than $\left(C-T_{1}\right)_{o}=1.82$ are barely detectable and have not been included in the estimate of the $r m s$ values (constrained to the $\left(C-T_{1}\right)$ range from 0.93 to 1.87 in what follows). Only 8 percent of the cluster population in this sample are redder than $\left(C-T_{1}\right)=1.87$ (making difficult the eventual detection of the reddest peaks).

The GCs sample within this region was analysed as a function of the $T_{1 o}$ magnitude aiming at detecting the $G C s$ magnitude range where, eventually, the colour pattern would be better defined.

Fig. 10 is the $G C C D$ corresponding to 588 clusters with $T_{1 o}=20.0$ to $T_{1 o}=22.0$ These objects probably include a fraction of $U C D$ objects overlapped with the brightest $G C s$ (e.g. Zhang et al. 2018). This sample is clearly dominated by blue $G C s$ and the pattern features are poorly defined $(r m s=0.034)$.

After adopting different ranges in the $T_{1 o}$ magnitudes, the best definition of the $T V P$ corresponds to clusters with $T_{1 o}=22.0$ to $T_{1 o}=23.7(\mathrm{rms}=0.019)$, shown in Fig. 11, and includes 1240 GCs. The [1.04] feature is clearly seen in this diagram.

Finally, the faintest sample, corresponding to $T_{1 o}=23.7$

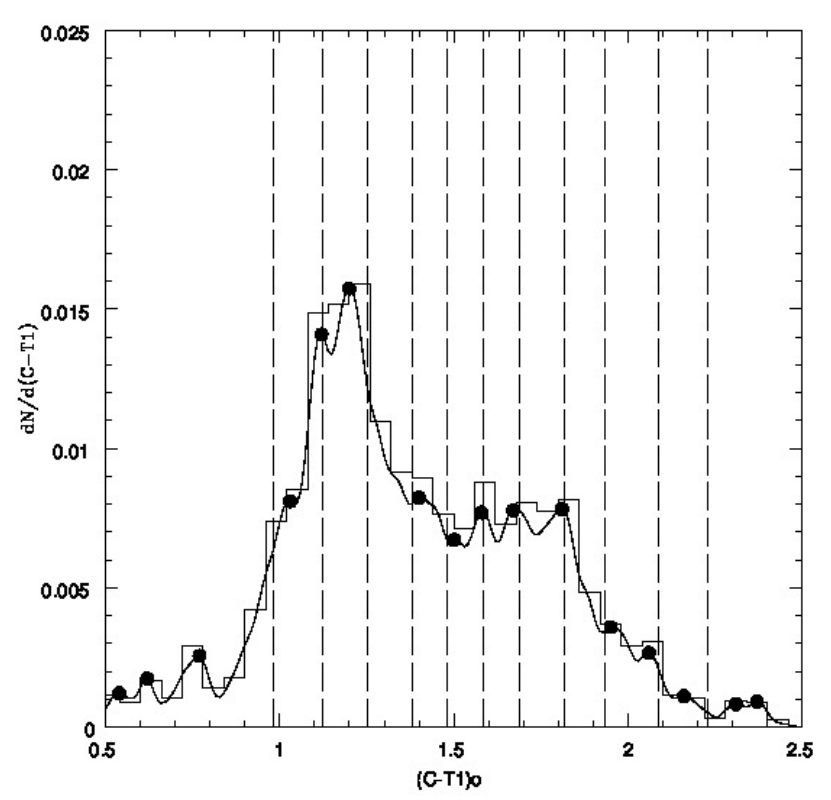

Figure 9. Globular cluster $\left(C-T_{1}\right)$ o colour distribution for 2048 objects with galactocentric radii from $90^{\prime \prime}$ to $240^{\prime \prime}$ and $T_{1 o}=21.0$ to 24.0 in $N G C$ 4486. Dots indicate the colour peaks detected by the $P F R$ routine. Vertical dashed lines indicate the $\left(C-T_{1}\right)$ components of the $T V P$.

to $T_{1 o}=24.00$, and including $242 G C s$, exhibits the $G C C D$ depicted in Fig. 12 ( $\mathrm{rms}=0.039)$. As expected, the TVP does not seem to be detectable in this sample as a consequence of the larger photometric errors as well as for the presence of an increasing number of field interlopers.

\subsection{The GCCD in NGC 4486 for galactocentric radii between $240^{\prime \prime}$ and $390^{\prime \prime}$.}

This region has an areal coverage of 80 percent in $F F G 07$ and its $G C C D$, displayed in Fig. 13, shows a single dominat blue peak and a rather disordered pattern with a large $r m s$ value of $0.035 \mathrm{mag}$. In principle, this result would indicate that the colour pattern is absent within that range of galactocentric radius.

A further test about this result was performed by analysing the GCCDs on galactocentric annuli $30^{\prime \prime}$ wide. On these annuli, the $P F R$ generates the catalogue that allows the determination of the relative frequency of the colour peaks (a treatment similar to that described in Section 3), as well as the colour shift that brings the colour pattern within a given galactocentric annulus into agreement with the TVP (by minimizing the colour $r m s$ values as explained before).

The number of $G C s$ within each annulus and the colour differences, are listed in Table 2. We note that the FFG07 photometry of GCs in NGC 4486 has a complete areal coverage in $R_{\text {gal }}$ between $90^{\prime \prime}$ and $390^{\prime \prime}$ and position angles ( $\mathrm{N}$ to E) from $90^{\circ}$ to $270^{\circ}$ (that corresponds to fifty percent of the total area) and that, in order to preserve an even coverage of the relative area of each annulus, the $P F R$ was run within these galactocentric limits. The GCs magnitude range was 


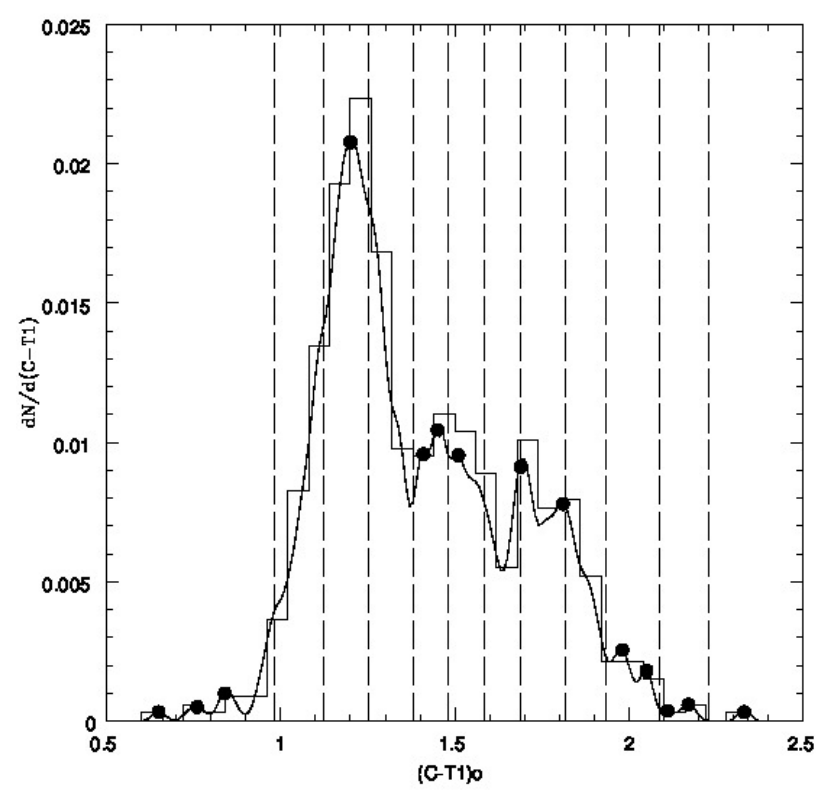

Figure 10. Globular cluster $\left(C-T_{1}\right)$ o colour distribution for 588 objects with galactocentric radii from $90^{\prime \prime}$ to $240^{\prime \prime}$ and $T_{1 o}=20.0$ to 22.0 , in $N G C$ 4486. Dots indicate the colour peaks detected by the $P F R$ routine. Vertical dashed lines indicate the $\left(C-T_{1}\right)$ components of the $T V P$.

Table 2. GCs Number of globular clusters and colour differences between the $T V P$ and the patterns found in different annular rings in NGC 4486.

\begin{tabular}{ccc}
\hline \hline Gal. range (arcsec) & $(g-z)_{o}$ colour diff. & N of GCs \\
\hline $90-120$ & +0.02 & 176 \\
$120-150$ & +0.02 & 149 \\
$150-180$ & 0.00 & 220 \\
$180-210$ & 0.00 & 180 \\
$210-240$ & 0.00 & 180 \\
$240-270$ & +0.04 & 179 \\
$270-300$ & +0.08 & 190 \\
$300-330$ & +0.08 & 190 \\
$330-360$ & +0.09 & 194 \\
$360-390$ & +0.10 & 195 \\
\hline
\end{tabular}

set from $T_{1 o}=19.2$ to 23.7 (corresponding to $g_{o} \approx 20.0$ to 24.5).

The relative frequency of the colour peaks based on 1853 $G C s$, without any colour shift, is displayed in Fig. 14. Eight peaks redder than $\left(C-T_{1}\right)_{o}=1.38$ have good coincidences with the $T V P$ colours ( $r m s= \pm 0.015)$. However, bluer peaks are in clear disagreement.

Fig. 15 exhibits the colour differences listed in Table 2 (with signs changed) plotted as a function of galactocentric radius. This figure indicates that the $T V P$ is detected with a consistent blueward trend as the galactocentric radius increases beyond $\approx 240^{\prime \prime}$. Thus, GCs in this galactocentric range seem to have a lower chemical abundance level compared to those in the inner region of the galaxy (see previous Section). Tentatively, this blueward trend can be identified with the GCs galactocentric colour gradient found by Forte

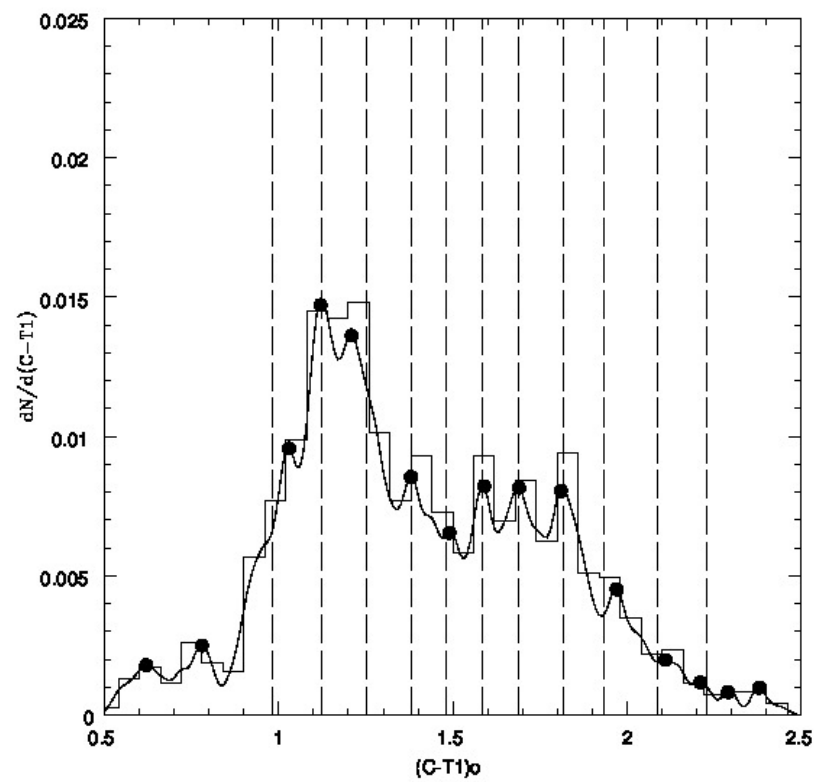

Figure 11. Globular cluster $\left(C-T_{1}\right)$ o colour distribution for 1240 objects with galactocentric radii from $90^{\prime \prime}$ to $240^{\prime \prime}$ and $T_{1 o}=22.0$ to 23.7 , in $N G C$ 4486. Dots indicate the colour peaks detected by the $P F R$ routine. Vertical dashed lines indicate the $\left(C-T_{1}\right)$ components of the $T V P$.

et al. (2012), indicated as a dashed line. This gradient corresponds to : $\left[\Delta\left(C-T_{1}\right) / \Delta\left(R_{\text {gal }}\right)\right]=2.6 \times 10^{-4} \mathrm{mag}$. $/{ }^{\prime \prime}$ (for 12 Gy old $G C s)$.

The adoption of the colour shifts listed in Table 2, leads to the relative frequency of the colour peaks depicted in Fig. 16, where, on one side, the peaks increase their contrast (compared to those in Fig. 14) and, on the other, leads to a nearly complete correspondence with the TVP colours(and a rms of 0.018).

Finally, the GCCDs displayed in Fig. 17 and Fig. 18, correspond to $T_{1 o}$ magnitude ranges of 20.0 to 24.0 and 22.0 to 23.7 , respectively. This last diagram, defined within the $T_{1}$ o magnitude range where the pattern is better defined, displays the feature at [1.04] and also, coincidences with all the TVP components, starting with the peak at [1.12].

\subsection{The GCCD in NGC 4486 for galactocentric radii between $90^{\prime \prime}$ and $390^{\prime \prime}$.}

The composite $G C C D s$, after combining the results discussed in the two previous subsections, and for two magnitude ranges $\left(T_{1 o}=20.0\right.$ to 24.0 and $T_{1 o}=22$ to 23.7$)$, are presented in Fig. 19 (rms =0.019) and Fig. 20 ( $r m s=0.017)$, respectively. Both diagrams display essentially the same colour patterns. This region covers an area about seven times larger than a typical $A C S$ frame and includes a contamination level by field interlopers between 10 and 15 percent at a limiting magnitude $T_{1}=23.7$ (see Section 4 ), i. e., 200 to 250 objects. These field interlopers may produce spurious peaks that eventually smear-out the TVP pattern.

The persistence of the $T V P$ as a function of spatial distribution is confirmed in Fig. 21 and Fig. 22, correspond- 


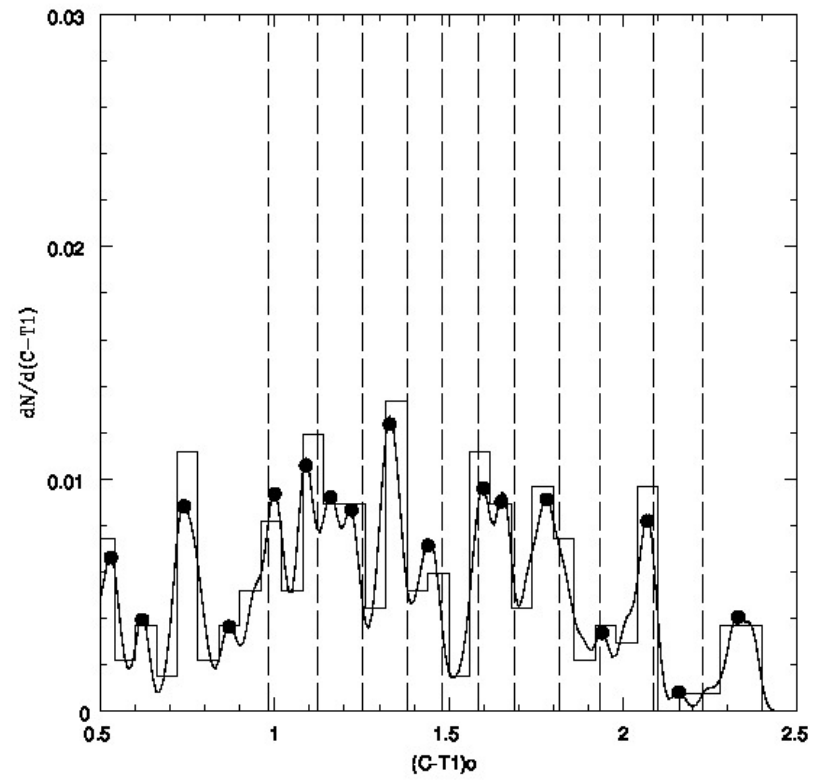

Figure 12. Globular cluster $\left(C-T_{1}\right) o$ colour distribution for 242 objects with galactocentric radii from $90^{\prime \prime}$ to $240^{\prime \prime}$ and $T_{1 o}=23.7$ to 24.0 , in $N G C 4486$. Dots indicate the colour peaks detected by the $P F R$ routine. Vertical dashed lines indicate the $\left(C-T_{1}\right)$ components of the $T V P$.

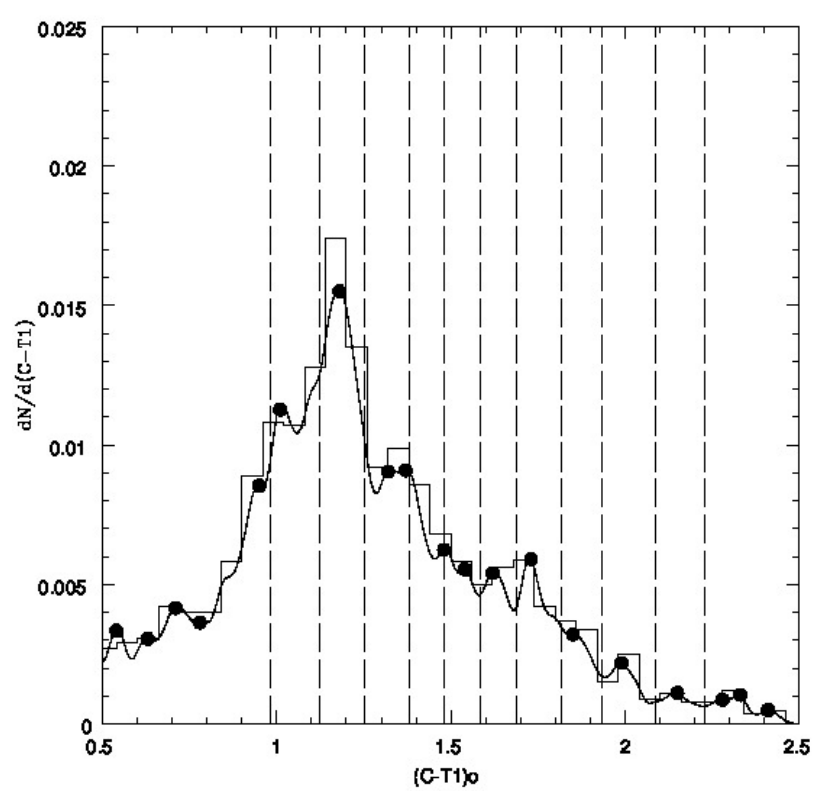

Figure 13. $\left(C-T_{1}\right) o G G C D$ for 1800 clusters with galactocentric radii from $240^{\prime \prime}$ to $390^{\prime \prime}$ and $T_{1 o}=20.0$ to 24.0 in NGC 4486. Dots are colour peaks found by the $P F R$. Vertical lines indicate the $T V P$ colours.

ing to the $G C C D s$ for objects with $T_{1 o}=22.0$ to 23.7 and position angles from $0^{\circ}$ to $180^{\circ} \quad(\mathrm{rms}=0.018)$ and $180^{\circ}$ to $360^{\circ} \quad(\mathrm{rms}=0.017)$, respectively.

On the other side, the behaviour of the TVP for different ranges of the $T_{1 o}$ magnitude, after adopting the colour shifts listed in Table 2, is displayed in Fig. A1 to Fig. A5.

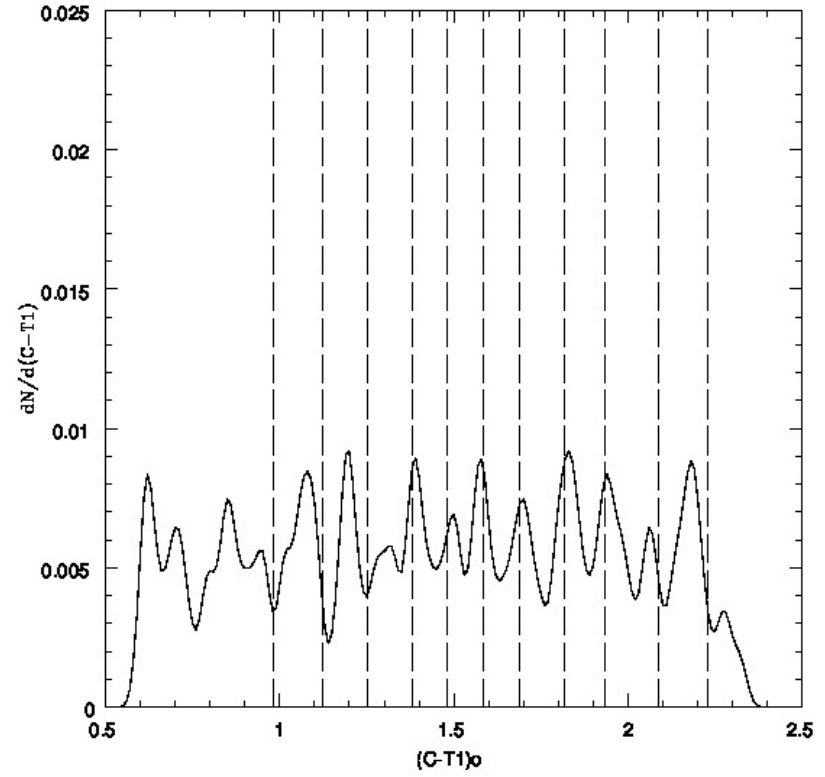

Figure 14. Relative frequency of $\left(C-T_{1}\right)_{o}$ colour peaks (without any colour shift) derived from data listed in Table 2. Vertical lines correspond to the $T V P$ colours. A disagreement between the $T V P$ colours is evident for peaks bluer than $\left(C-T_{1}\right)_{o}=1.38$.

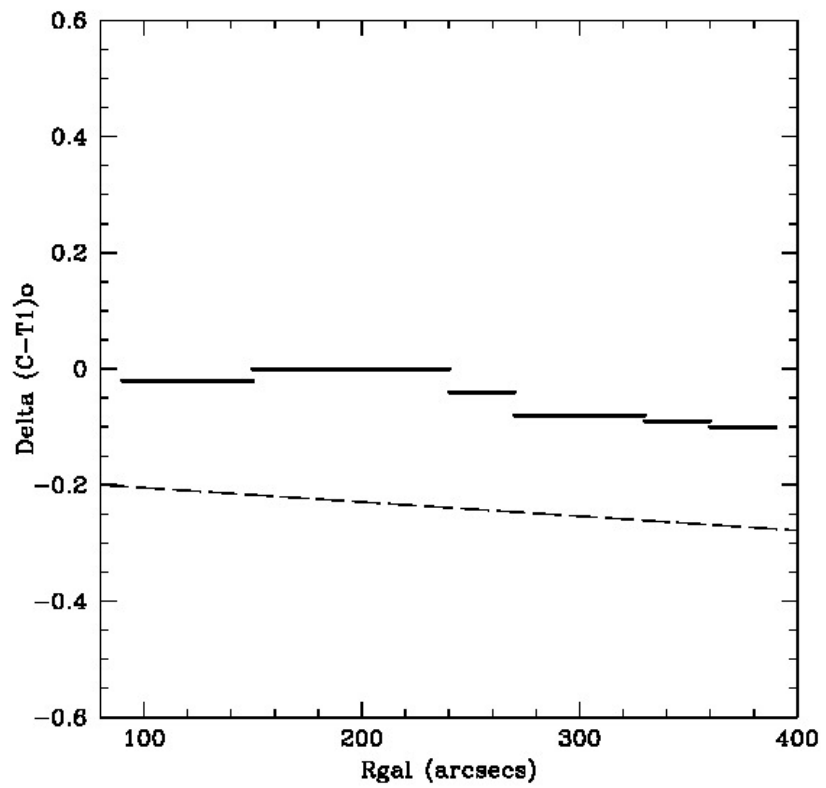

Figure 15. $\left(C-T_{1}\right)_{o}$ colour difference between the colour pattern observed within a given galactocentric range, indicated by the horizontal lines, and the TVP colours. The dashed line (arbitrarily shifted downwards) corresponds to the $G C$ colour gradient mentioned in the text.

The first of these diagrams corresponds to 946 objects with $T_{1 o}$ from 20.0 to 22.0. This GCCD has a dominant and single blue peak $\left[\left(C-T_{1}\right)_{o}=1.25\right]$. As noted previously, some degree of contamination by ultra compact objects can be expected in this range of magnitude. In turn, Fig. A2 $\left(T_{1 o}=\right.$ 22.0 to $22.7 ; 816$ objects), Fig. A3 ( $T_{1 o}=22.7$ to $23.25 ; 777$ 


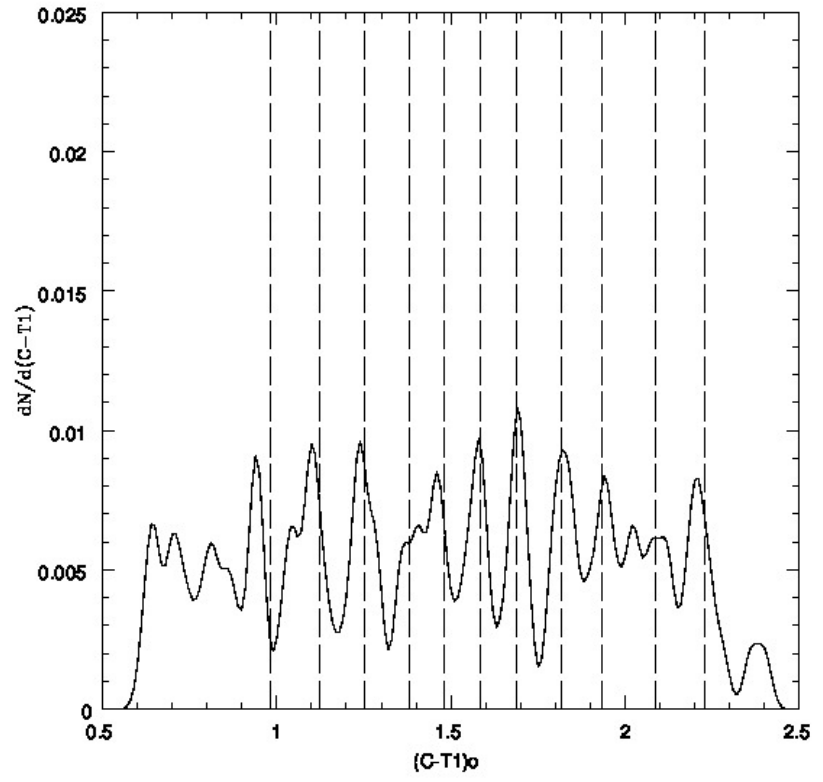

Figure 16. Relative frequency of $\left(C-T_{1}\right)_{o}$ colour peaks derived from data listed in Table 2. Vertical lines correspond to the TVP colours. The $G C$ colours have been shifted according to the values indicated in the text.

objects), and Fig. A4 ( $T_{1 o}=23.25$ to $23.7 ; 785$ objects), show the presence of seven to eight $T V P$ features. These ranges in $T_{1}$ magnitude were set in order to include an approximately similar number of cluster candidates $(\approx 800$ objects). The blue peak at (1.04) is seen in both Fig. A2 and Fig. A3.

Fig. A5 corresponds to 1212 objects with $T_{1 o}$ from 23.7 to 24.5. In this sample the $T V P$ is not detectable as a consequence of the larger photometric errors as well as the presumably increasing number of field interlopers.

In turn, Fig. 23 shows the smoothed colour-magnitude diagram (similar to that introduced in Fig. 6). In this diagram, that includes $3324 \mathrm{GCs}$, we set a magnitude cut off at $T_{1 o}=23.70$, as fainter clusters do not show the $T V P$ (see Fig. 12). The contour of the assymetric convolving kernel (0.25 mag. in $T_{1 o}$ and $0.019 \mathrm{mag}$. in $\left(C-T_{1}\right)$ is displayed at upper right. Again, as in Fig. 6, the $G C C D$ seems highly structured and in concordance with the $T V P$ colours.

Besides the two dominant blue peaks, at [1.12] and [1.25], Fig. 23 suggests the presence of several bluer components, being the feature at [1.04] the most prominent one.

\section{THE INTEGRATED GC LUMINOSITY FUNCTIONS OF THE TVP COMPONENTS IN NGC 4486.}

The integrated luminosity function ( $L F$ in what follows) corresponding to $2931 \mathrm{GCs}$ with $\left(C-T_{1}\right)$ colours from 0.93 to 2.28 (and normalized by the total number of clusters) is displayed in Fig. A6. As expected, this distribution can be properly represented with a Gaussian function characterized by a mean magnitude $T_{1 o}=23.3$ and a dispersion $\sigma_{T_{1}}=1.3$ mag, also shown in this and in figures A7 to A16. The turnover magnitude is consistent with that derived by Villegas

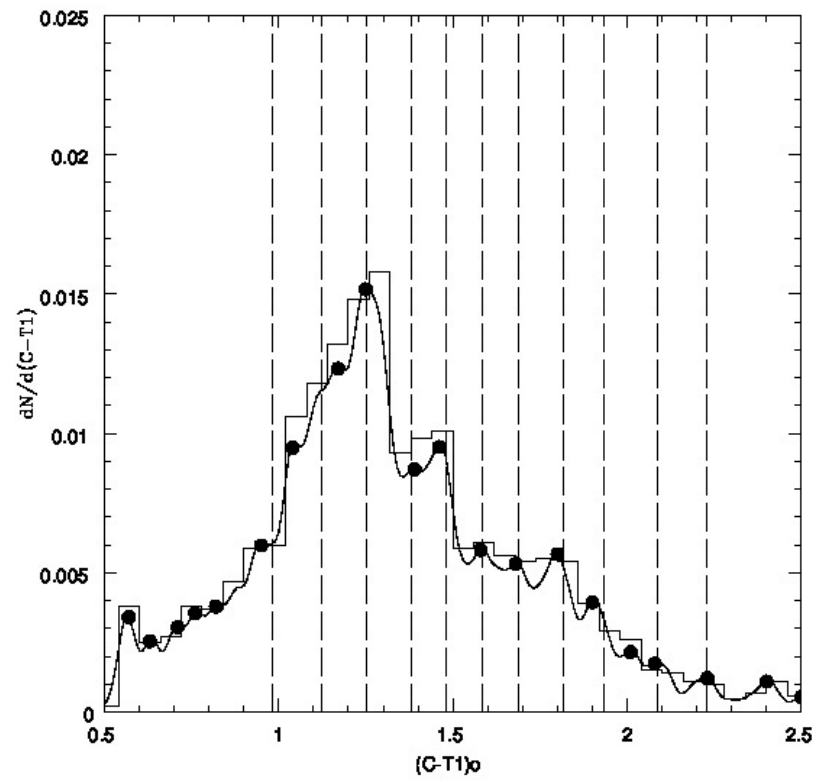

Figure 17. Globular cluster $\left(C-T_{1}\right)$ o colour distribution for 1800 objects with galactocentric radii from $240^{\prime \prime}$ to $390^{\prime \prime}$ and $T_{1 o}=20.0$ to 24.0 in $N G C$ 4486. Dots indicate the colour peaks detected by the $P F R$ routine. Vertical dashed lines indicate the $\left(C-T_{1}\right)$ components of the TVP. Colours have been corrected according to the values listed in Table 2 .

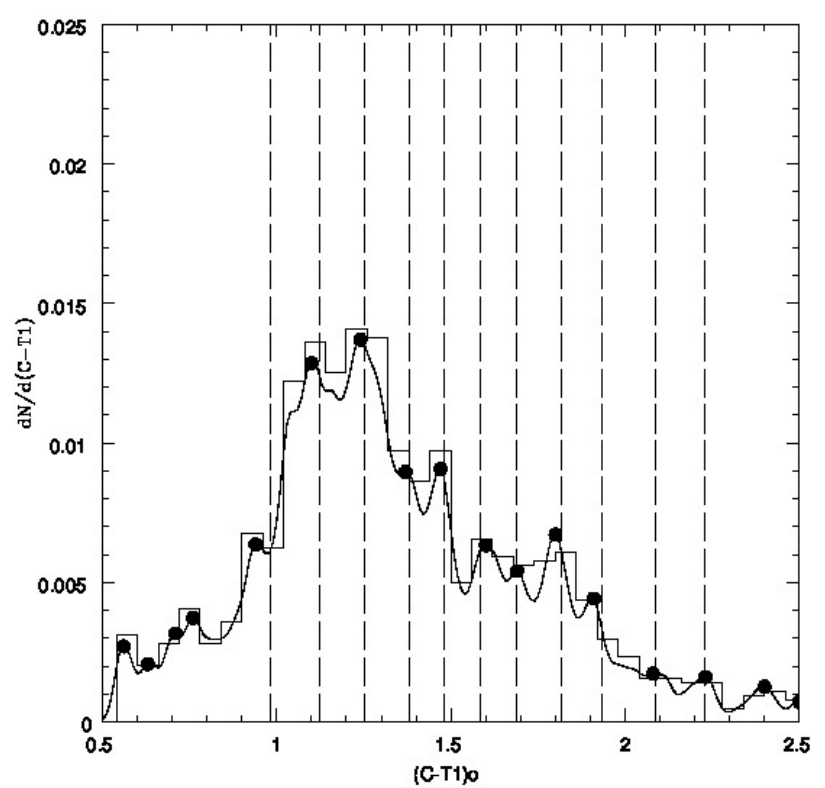

Figure 18. Globular cluster $\left(C-T_{1}\right)$ o colour distribution for 1100 objects with galactocentric radii from $240^{\prime \prime}$ to $390^{\prime \prime}$ and $T_{1 o}=22.0$ to 23.7, in $N G C$ 4486. Dots indicate the colour peaks detected by the $P F R$ routine. Vertical dashed lines indicate the $\left(C-T_{1}\right)$ components of the TVP. Colours have been corrected according to the values listed in Table 2 . 


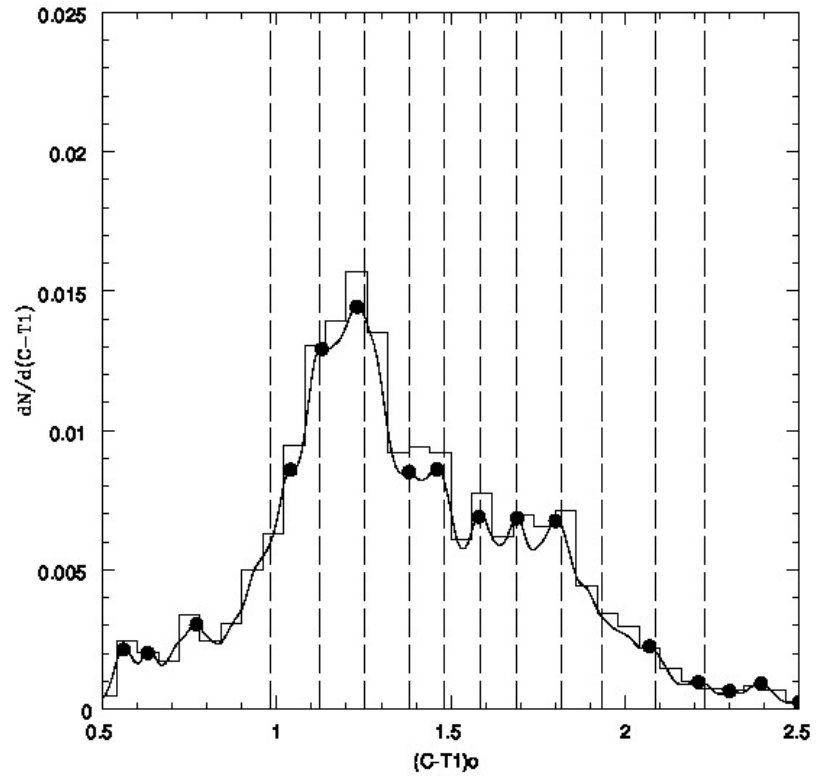

Figure 19. Globular cluster $\left(C-T_{1}\right) o$ colour distribution for 3848 objects with galactocentric radii from $90^{\prime \prime}$ to $390^{\prime \prime}$ and $T_{1 o}=20.0$ to 24.0 in $N G C 4486$. Dots indicate the colour peaks detected by the $P F R$ routine. Vertical dashed lines indicate the $\left(C-T_{1}\right)$ components of the $T V P$. Colours have been corrected as explained in the text.

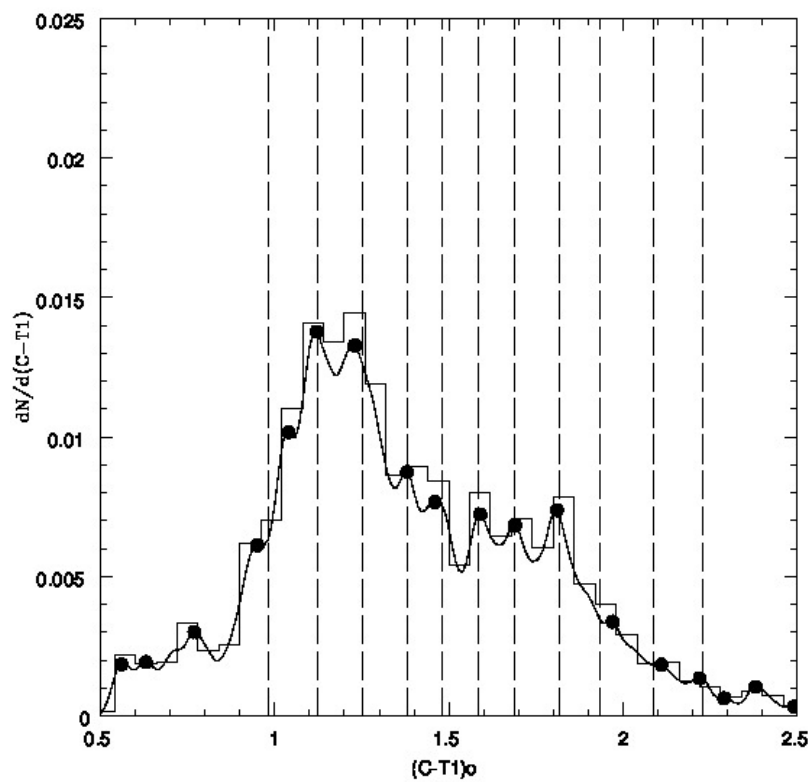

Figure 20. Globular cluster $\left(C-T_{1}\right) o$ colour distribution for 2389 objects with galactocentric radii from $90^{\prime \prime}$ to $390^{\prime \prime}$ and $T_{1 o}=22$ to 23.7 in $N G C 4486$. Dots indicate the colour peaks detected by the $P F R$ routine. Vertical dashed lines indicate the $\left(C-T_{1}\right)$ components of the $T V P$. Colours have been corrected as explained in the text.

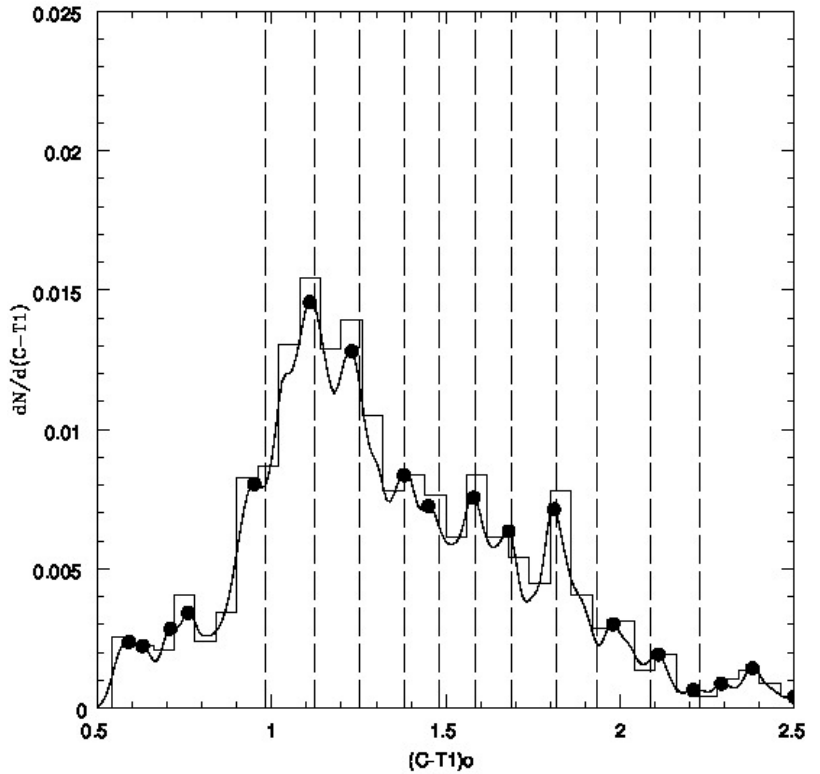

Figure 21. Globular cluster $\left(C-T_{1}\right)$ o colour distribution for 1201 objects with galactocentric radii from $90^{\prime \prime}$ to $390^{\prime \prime}$ and $T_{1 o}=22$ to 23.7 and position angles from $0^{\circ}$ to $180^{\circ}$ in $N G C$ 4486. Dots indicate the colour peaks detected by the $P F R$ routine. Vertical dashed lines indicate the $\left(C-T_{1}\right)$ components of the TVP. Colours have been corrected as explained in the text.

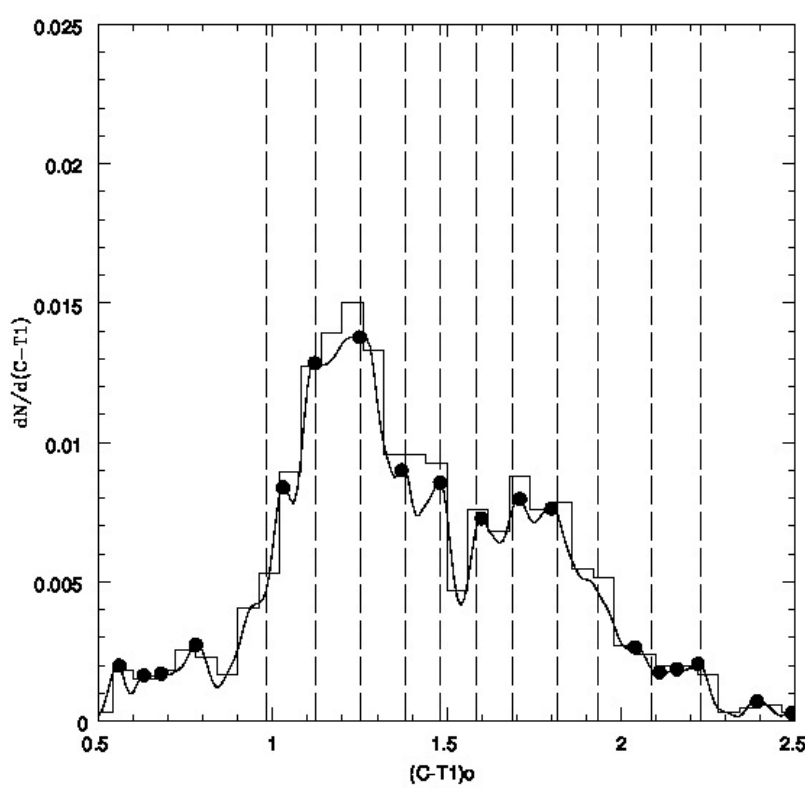

Figure 22. Globular cluster $\left(C-T_{1}\right) o$ colour distribution for 1181 objects with galactocentric radii from $90^{\prime \prime}$ to $390^{\prime \prime}$ and $T_{1 o}=22$ to 23.7, and position angles from $180^{\circ}$ to $360^{\circ}$ in $N G C$ 4486. Dots indicate the colour peaks detected by the PFR routine. Vertical dashed lines indicate the $\left(C-T_{1}\right)$ components of the TVP. Colours have been corrected as explained in the text. 


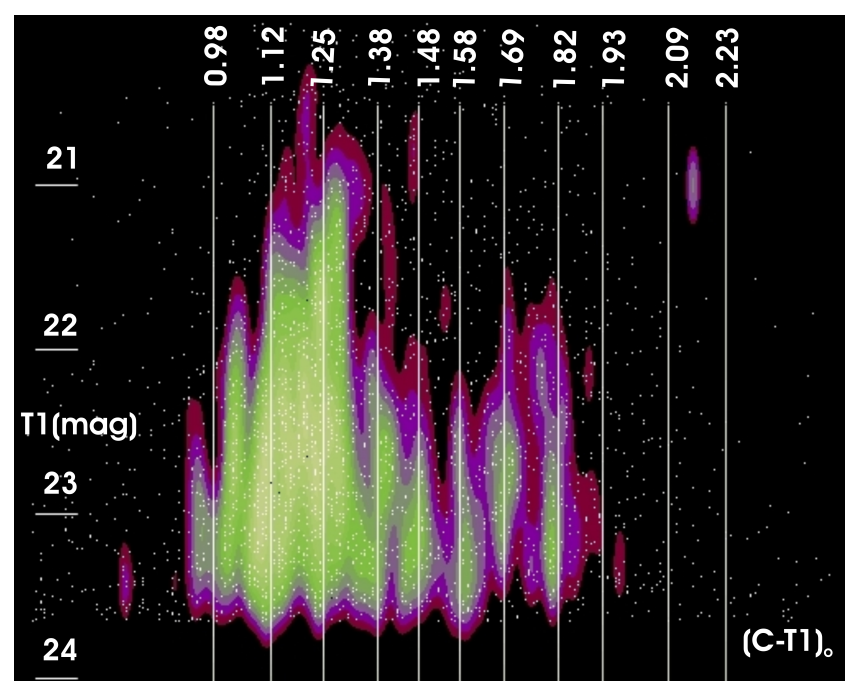

Figure 23. Smoothed colour-magnitude diagram for 3324 GCs with galactocentric radii from $90^{\prime \prime}$ to $390^{\prime \prime}$ and $T_{1 o}=20.0$ to 23.7 , and adopting the colour shifts listed in Table 2 . The vertical lines correspond to the TVP colours. White dots represent individual GCs. The contour of the convolving kernel is shown at upper right.

et al. (2010). All these diagrams include the size of the convolving kernel (adopted for Figs. 6 and 23) which is about 5 percent of the total range in the $T_{1 o}$ magnitude.

In order to explore the nature of the colour peaks that define the $T V P$, we analysed the characteristics of both the distribution of the GCs on the sky and of their integrated $T_{1 o}$ luminosity functions. This analysis was performed adopting $0.1 \mathrm{mag}$. colour windows centered at the detectable TVP components and also at the [1.04] feature.

The nature of the [1.04] colour peak is intriguing. This feature, and in general objects bluer than $\left(C-T_{1}\right)=1.17$, share similar and very shallow spatial distributions. Their $L F s$, shown in Fig. A7, A8, and A9 are also similar and steeper than the Gaussian that gives a good representation of the whole $G C$ sample. It is tempting to suggest that, at least a fraction, of this population of $G C$ candidates are in fact members of the so called "Virgo Intra Cluster GC population" (e.g. Ko et al. 2017) although this cannot be proven just on photometric data.

The remaining $T V P$ components show a variable degree of concentration towards the galaxy centre, that increases with redder colours.

In general, the LFs shown in Fig. A7 to A16 are broadly consistent with the Gaussian approximation shown in Fig. A6 (i.e., the observed counts are within $\approx 3$ times the expected Poissonian deviations). However, the clarification of the nature of some "clumps" in these figures will require further photometric and spectroscopic data.

\section{CONCLUSIONS}

In summary, this paper reports that:

(i) A revision of the TVP colours, after increasing the $G C s$ sample by a factor of three, compared to that discussed in F2017, confirms that the pattern is a solid feature detectable in the 27 brightest Virgo galaxies, including 12 giant galaxies not considered in that work.

(ii) After grouping the $G C s$ in these 27 galaxies in three independent samples, each with approximately the same number of objects ( $\approx 1400$ clusters), the $G C C D s$ exhibit between six and eight colour peaks that are compatible with the components of the TVP presented in F2017.

(iii) At least seven of the $T V P$ components are readily detectable in $N G C 4486$, for $G C s$ having $A C S$ photometry, and $R_{\text {gal }}$ from $40^{\prime \prime}$ to $90^{\prime \prime}$.

(iv) In turn, a new analysis of the photometry in the $C$ and $T_{1 o}$ filters (Washington system) presented in FFG07 for $G C s$ in $N G C 4486$, indicates that the $T V P$ is detectable on the $\left(C-T_{1}\right)_{o}$ colours in a galactocentric range $R_{\text {gal }}$ from $90^{\prime \prime}$ to $240^{\prime \prime}$ without requiring any colour shift. This $G C$ sample includes some 2000 objects and does not overlap with the $A C S$ clusters of the inner regions of the galaxy.

(v) Clusters with $R_{\text {gal }}$ from $240^{\prime \prime}$ to $390^{\prime \prime}$ require redward colour shifts in order to match the colour patterns in different galactocentric annuli in $N G C 4486$ to the $T V P$ colours . As a whole, these shifts are compatible with the GCs galactocentric colour gradient determined in Forte et al. (2012) and suggest that these clusters have lower chemical abundances than those that defined the TVP.

(vi) The adoption of a distance modulus of 31.1 for the Virgo cluster (e.g. Tonry et al. 2001), then indicates that the $T V P$ is detectable, at least, in a galactocentric range of 3 to $30 \mathrm{Kpc}\left(40^{\prime \prime}\right.$ to $\left.390^{\prime \prime}\right)$.

(vii) The presence of the $T V P$ is not only detectable at different galactocentric radii but also when the GCs sample in the whole galactocentric range from $90^{\prime \prime}$ to $390^{\prime \prime}$ is split as a function of position angle (e.g., from $0^{\circ}$ to $180^{\circ}$ and from $180^{\circ}$ to $360^{\circ}$ ).

(viii) The detection of the $T V P$ on top of the clusters colour gradient in $N G C 4486$, suggests that these features were eventually imprinted during the dissipative collapse stage of the formation of the galaxy.

(ix) Globally, the TVP components are detectable on the two cluster (blue and red) sub populations that define the broad GCs colour "bi-modality" in NGC 4486 (see, for example, FFG07).

(x) The presence of the TVP pattern on both the $(g-z) o$ and $\left(C-T_{1}\right)_{o}$ colours, and in different $G C s$ samples, gives a strong argument to reject instrumental effects as the origin of that pattern. Another argument in the same sense is provided by the fact that the $T V P$ is not evident neither for $G C s$ belonging to galaxies fainter than $M_{g}=-18.2$, nor for clusters inside a galactocentric radius of $40^{\prime \prime}$ in giant galaxies. Other causes, as contamination by field interlopers or statistical fluctuations were already rejected in F2017.

(xi) The existence of two blue GCs components, with $(g-$ $z) o=0.85$ and 0.95 respectively, and most evident for clusters in moderately bright galaxies (i.e. $M_{g}=-20.2$ to -19.2 ), is consistent with a similar situation detectable in the outer regions of $N G C 4486$ (with corresponding colour peaks at $\left(C-T_{1}\right) o=1.12$ and 1.25$)$.

(xii) Regarding the eventual "universality" of the TVP (and as noted in F2017), each $G C$ system might have clusters that are part of its own history (including galaxy mergers, etc.) as well as other clusters that eventually formed in connection with the $T V P$. Because of this, the $T V P$ detectability would depend on each particular case. However, 
the pattern emerges even in massive galaxies with presumably complex histories, as $N G C 4486$, discussed in this paper.

A tentative explanation for the colour patterns, given in F2017, suggests that an external mechanism has been able to modulate the $G C s$ formation over supra-galactic spatial scales in a kind of viral process, at high redshifts, and following what seems to be a temporal sequence. If this is the situation, the effect of such a putative phenomenon might also have left its signature early on the diffuse stellar population of these galaxies.

The lack of substantial evidence supporting a given candidate mechanism ( $A G N$ activity ? sub-cluster merger events ?) that could be responsible for the GCs colour pattern, defines the current situation as a supra - galactic conundrum ("...a question or problem having only a conjectural answer"; Merriam-Webster dictionary) that deserves further exploration.

\section{ACKNOWLEDGEMENTS}

This work was funded with grants from Consejo Nacional de Investigaciones Científicas y Técnicas de la República Argentina (CONICET). J.C.F. thanks Dr. Gustavo Corach for his hospitality at the IAM-CONICET. D.G. gratefully acknowledges support from the Chilean Centro de Excelencia en Astrofísica y Tecnologías Afines (CATA) BASAL grant AFB-170002, and also the financial support from the Dirección de Investigación y Desarrollo de la Universidad de La Serena through the Programa de Incentivo a la Investigación de Académicos (PIA-DIDULS).

\section{REFERENCES}

Brodie J. P., Strader J., 2006, ARA\&A, 44, 193

Escudero C. G., Faifer F. R., Smith Castelli A. V., Forte J. C., Sesto L. A., González N. M., Scalia M. C., 2018, MNRAS, 474, 4302

Ferrarese L., et al., 2006, ApJS, 164, 334

Forte J. C., 2017, MNRAS, 468, 3917

Forte J. C., Faifer F., Geisler D., 2007, MNRAS, 382, 1947

Forte J. C., Vega E. I., Faifer F., 2012, MNRAS, 421, 635

Forte J. C., Faifer F. R., Vega E. I., Bassino L. P., Smith Castelli A. V., Cellone S. A., Geisler D., 2013, MNRAS, 431, 1405

Harris W. E., Ciccone S. M., Eadie G. M., Gnedin O. Y., Geisler D., Rothberg B., Bailin J., 2017, ApJ, 835, 101

Jordán A., et al., 2009, ApJS, 180, 54

Jordán A., Peng E. W., Blakeslee J. P., Côté P., Eyheramendy S., Ferrarese L., 2015, ApJS, 221, 13

Ko Y., et al., 2017, ApJ, 835, 212

Schlafly E. F., Finkbeiner D. P., 2011, ApJ, 737, 103

Sesto L. A., Faifer F. R., Forte J. C., 2016, MNRAS, 461, 4260

Tamura N., Sharples R. M., Arimoto N., Onodera M., Ohta K., Yamada Y., 2006, MNRAS, 373, 601

Tonry J. L., Dressler A., Blakeslee J. P., Ajhar E. A., Fletcher A. B., Luppino G. A., Metzger M. R., Moore C. B., 2001, ApJ, 546, 681

Villegas D., et al., 2010, ApJ, 717, 603

Zhang H.-X., et al., 2018, ApJ, 858, 37
This paper has been typeset from a $\mathrm{T}_{\mathrm{EX}} \mathrm{X} / \mathrm{ATEX}$ file prepared by the author.

APPENDIX A: ADDITIONAL DIAGRAMS. 


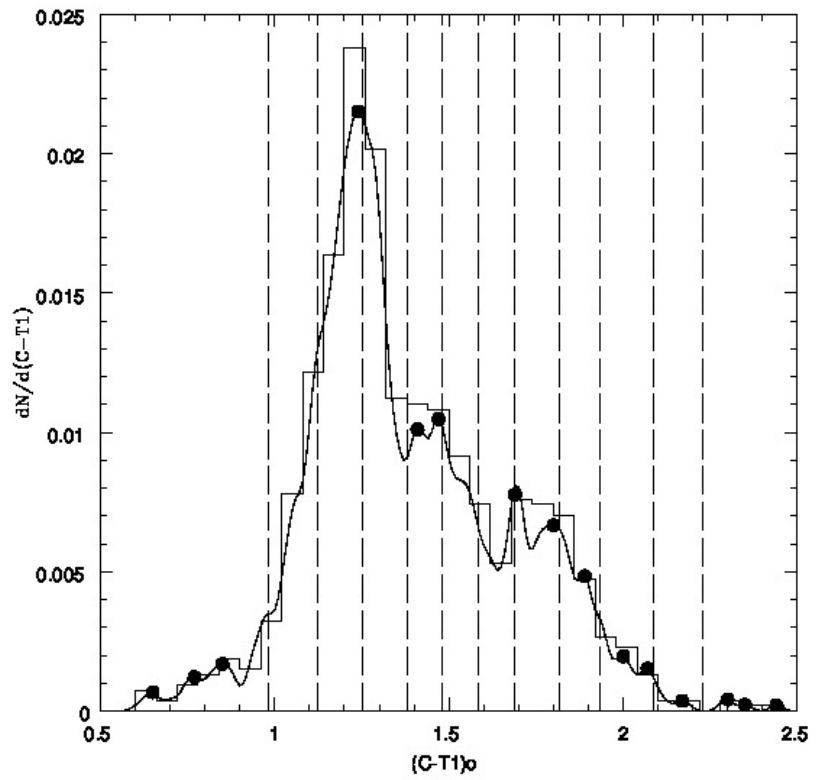

Figure A1. Globular cluster $\left(C-T_{1}\right)$ o colour distribution for 946 objects with galactocentric radii from $90^{\prime \prime}$ to $390^{\prime \prime}$ and $T_{1 o}=20.0$ to 22.0 in $N G C 4486$. Dots indicate the colour peaks detected by the $P F R$ routine. Vertical dashed lines indicate the $\left(C-T_{1}\right)$ components of the $T V P$. Colours have been corrected as explained in the text.

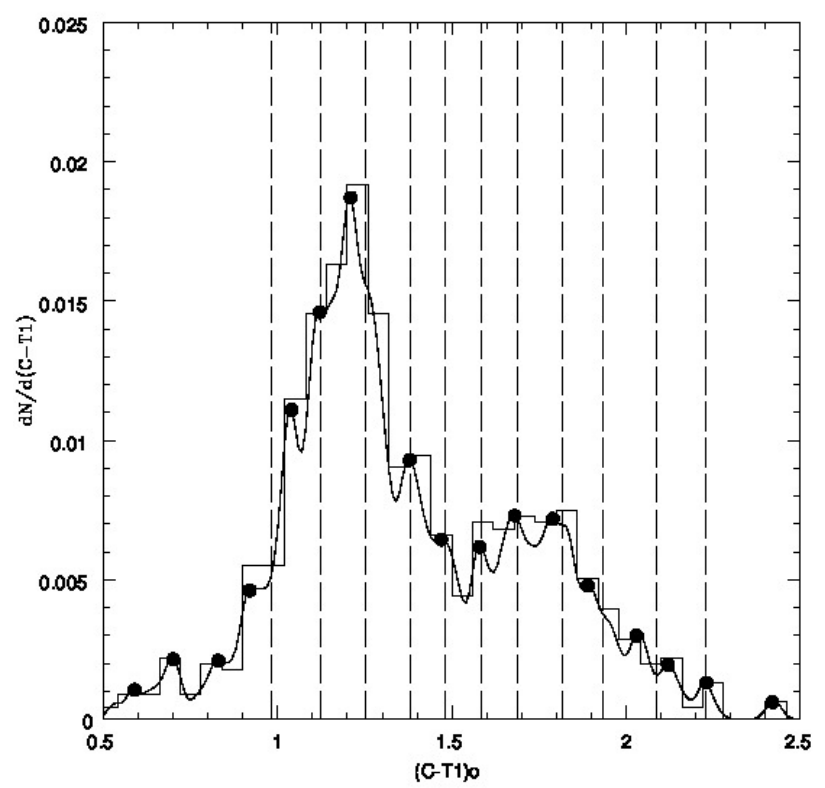

Figure A2. Similar to Fig. A1 for 816 objects with $T_{1 o}=22.00$ to 22.70 .

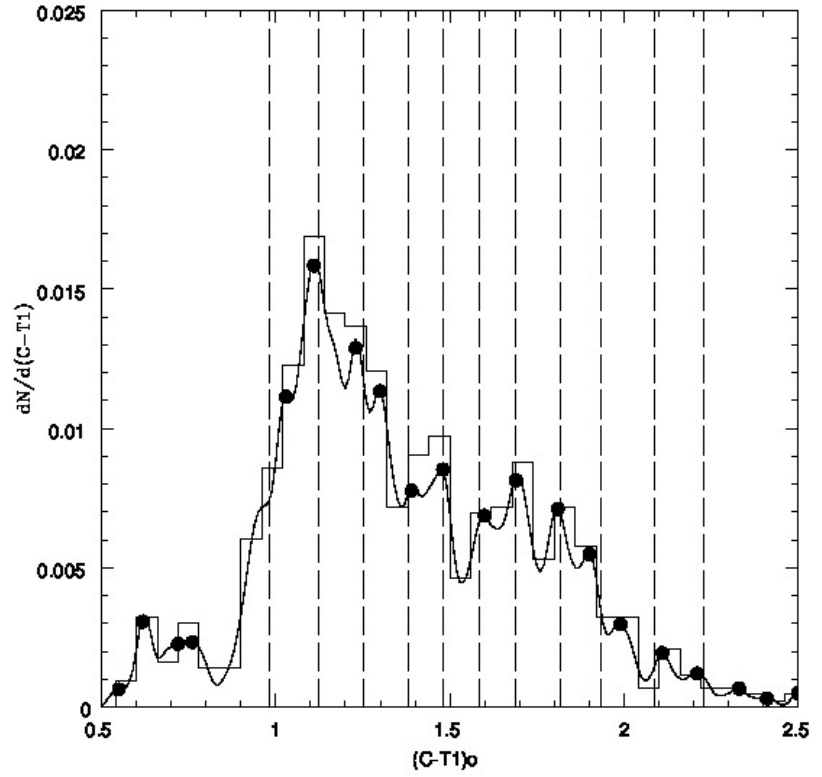

Figure A3. Similar to Fig. A1 for 777 objects with $T_{1 o}=22.70$ to 23.25 .

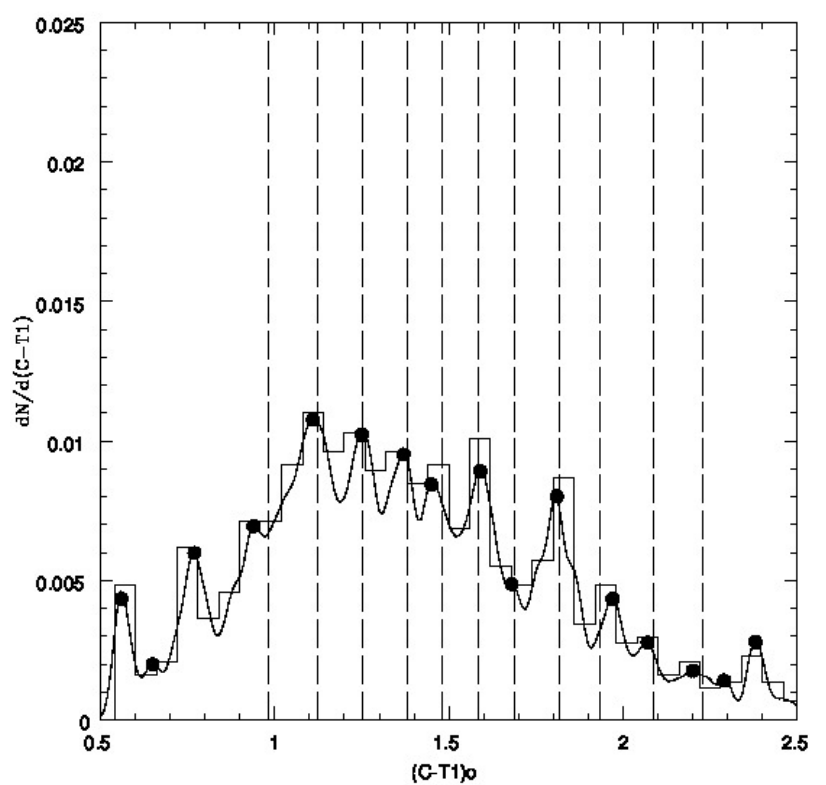

Figure A4. Similar to Fig. A1 for 785 objects with $T_{1 o}=23.25$ to 23.70 . 


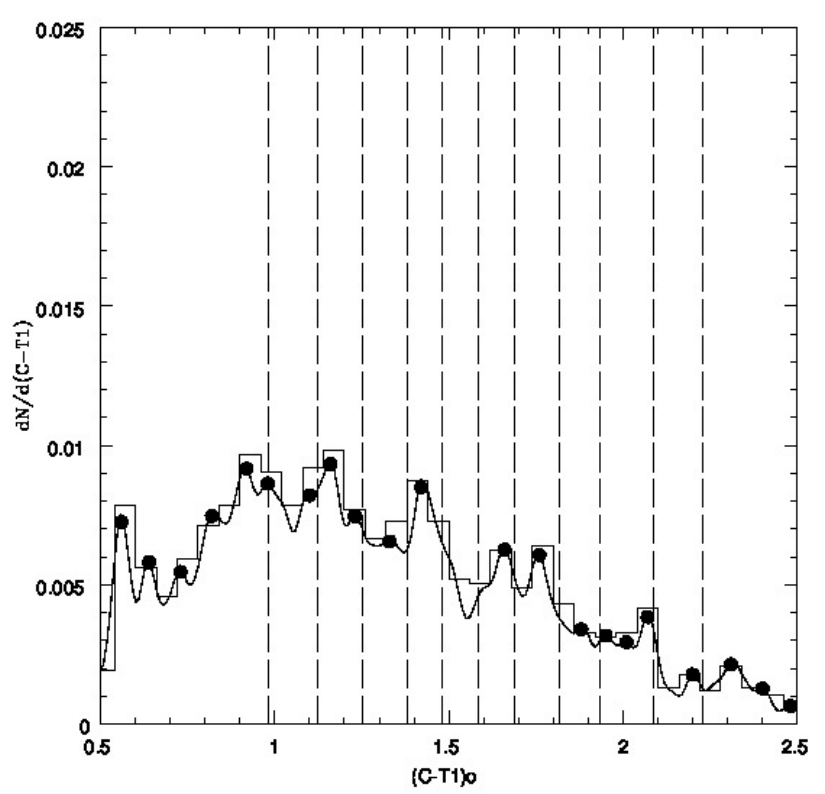

Figure A5. Similar to Fig. A1 for 1212 objects with $T_{1 o}=23.70$ to 24.50 .

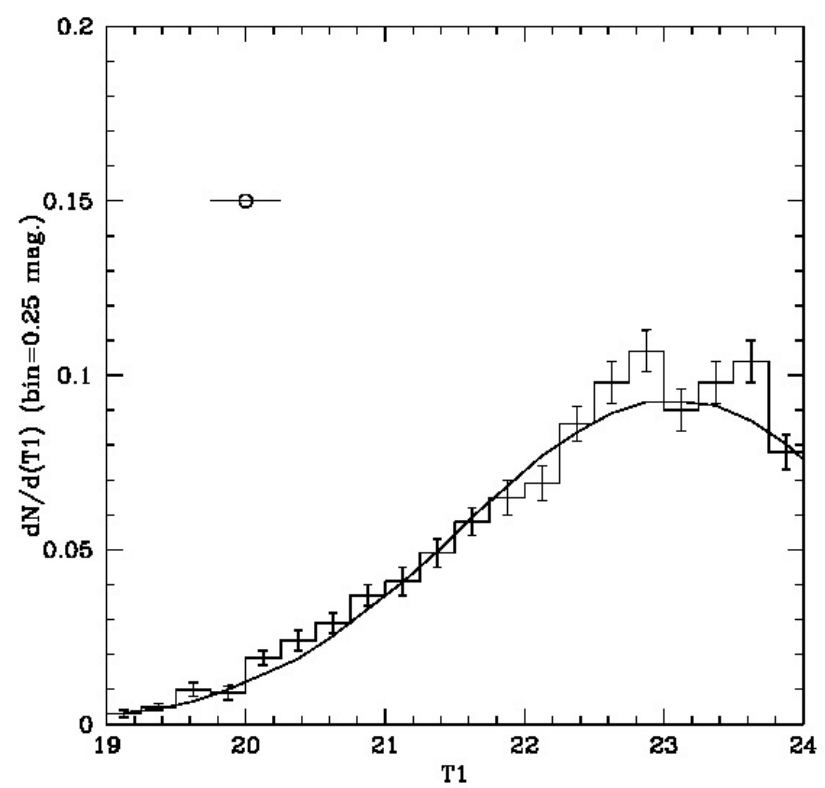

Figure A6. Integrated GCs luminosity function for 2782 GC candidates with galactocentric radii from $90^{\prime \prime}$ to $390^{\prime \prime}$ and $\left(C-T_{1}\right) o$ colours from 0.93 to 2.28 . The solid line corresponds to a reference Gaussian fit (see text). Vertical bars indicate the counting uncertainties (see text). The horizontal bar at upper left shows the size of the convolving kernel used in Figs. 6 and 23.

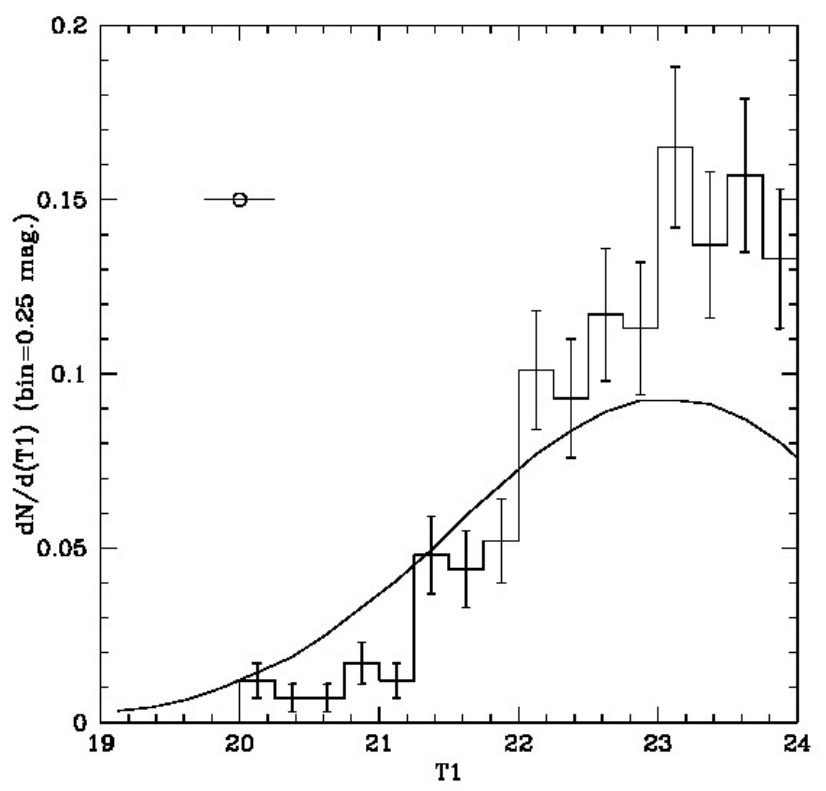

Figure A7. Similar to Fig. A6 but for 275 GC candidates with $\left(C-T_{1}\right) o$ colours from 0.93 to 1.03 .

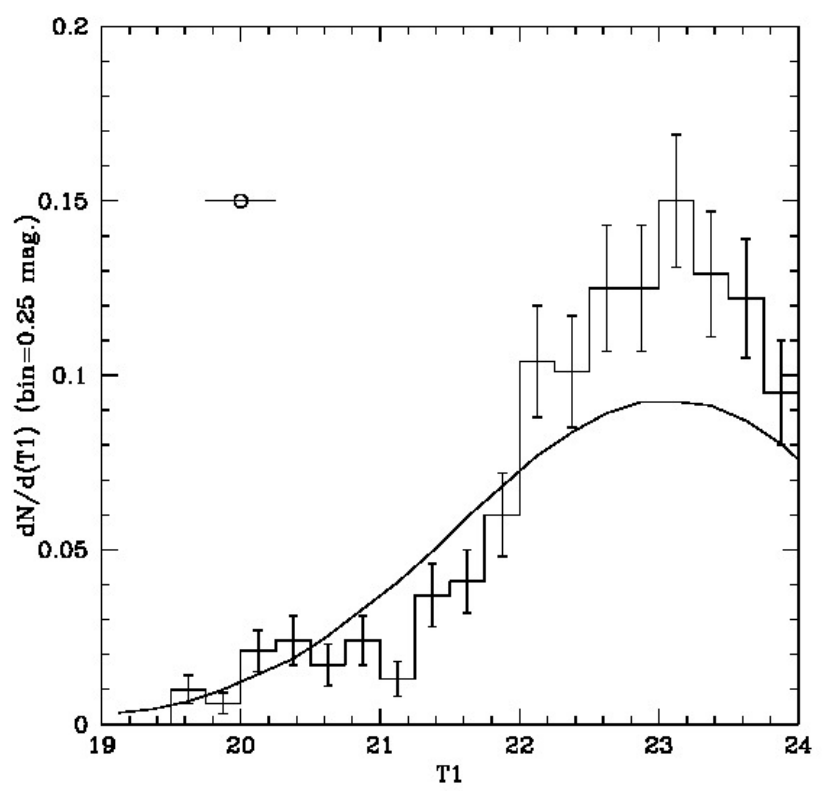

Figure A8. Similar to Fig. A6 but for 352 GC candidates with $\left(C-T_{1}\right) o$ colours from 0.99 to 1.09 . 


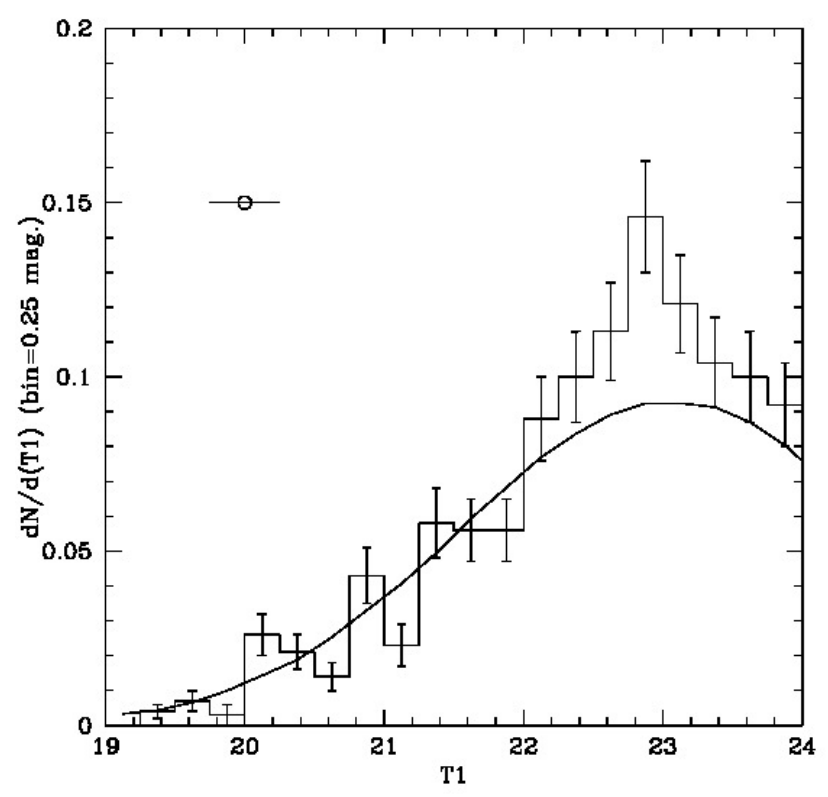

Figure A9. Similar to Fig. A6 but for 516 GC candidates with $\left(C-T_{1}\right) o$ colours from 1.07 to 1.17 .

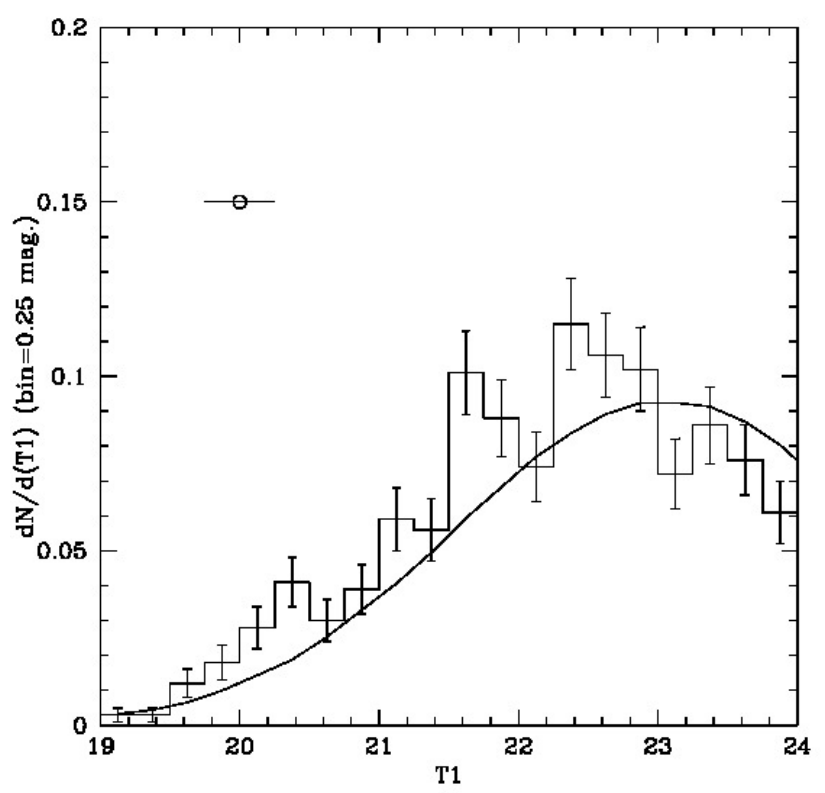

Figure A10. Similar to Fig. A6 but for 599 GC candidates with $\left(C-T_{1}\right) o$ colours from 1.20 to 1.30 .

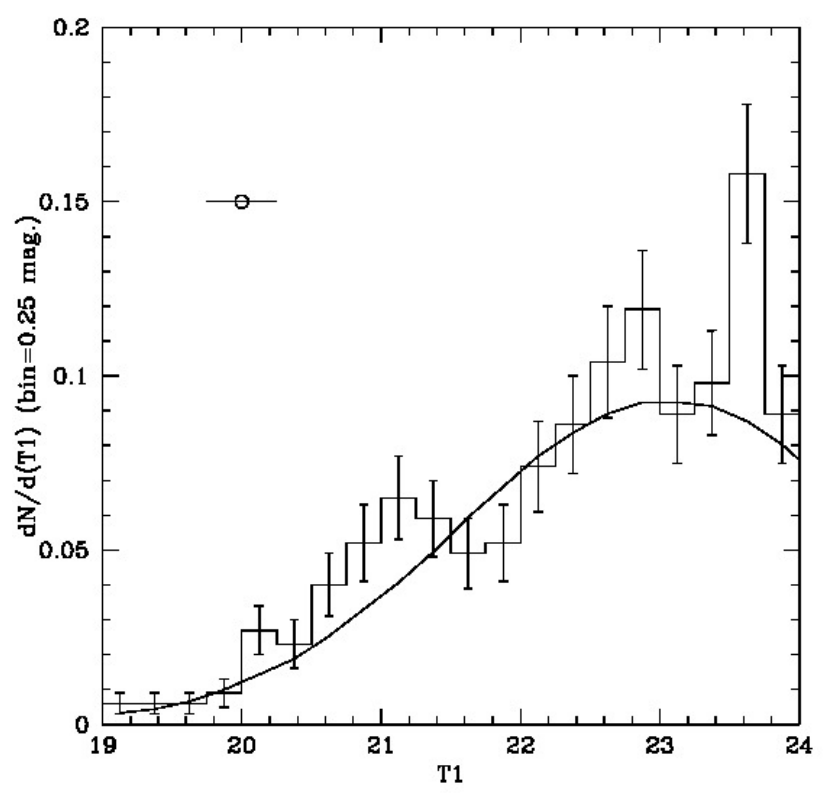

Figure A11. Similar to Fig. A6 but for $361 \mathrm{GC}$ candidates with $\left(C-T_{1}\right) o$ colours from 1.33 to 1.43 .

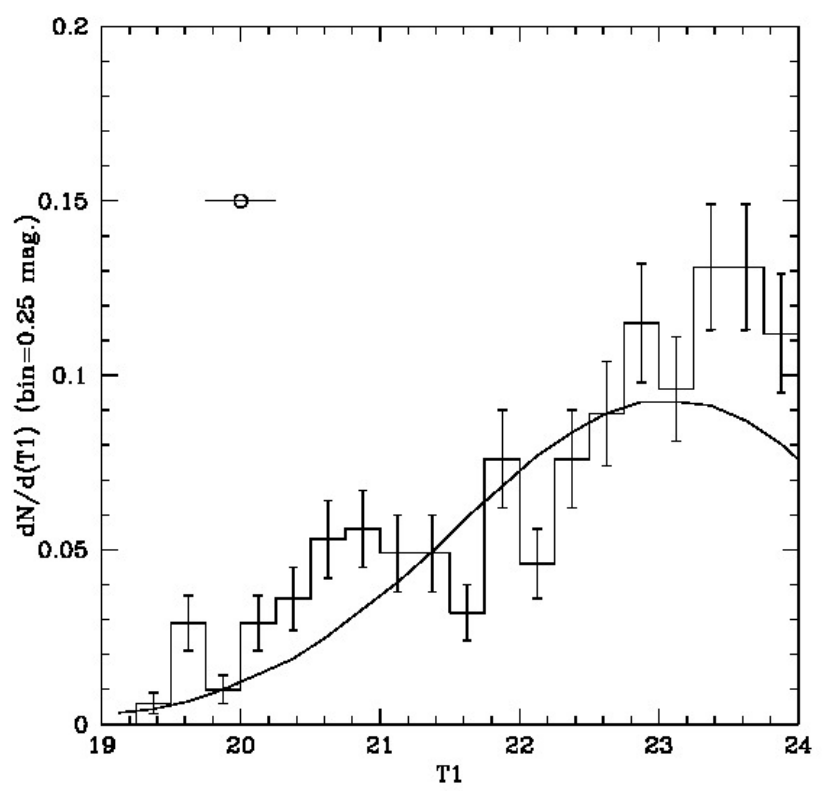

Figure A12. Similar to Fig. A6 but for 337 GC candidates with $\left(C-T_{1}\right) o$ colours from 1.43 to 1.53 . 
Forte et al.

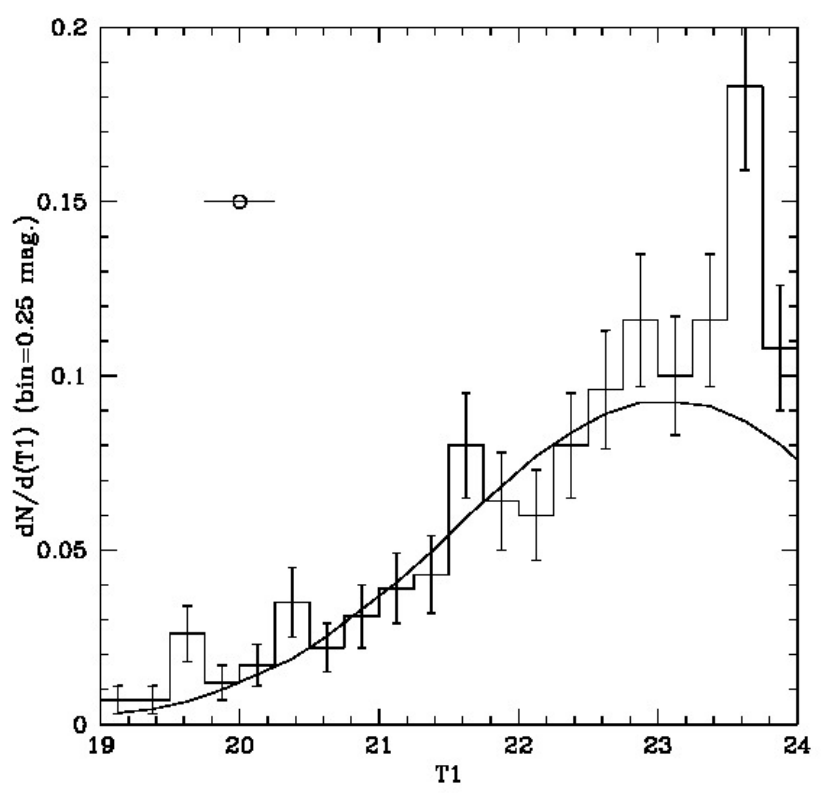

Figure A13. Similar to Fig. A6 but for 277 GC candidates with $\left(C-T_{1}\right) o$ colours from 1.53 to 1.63 .

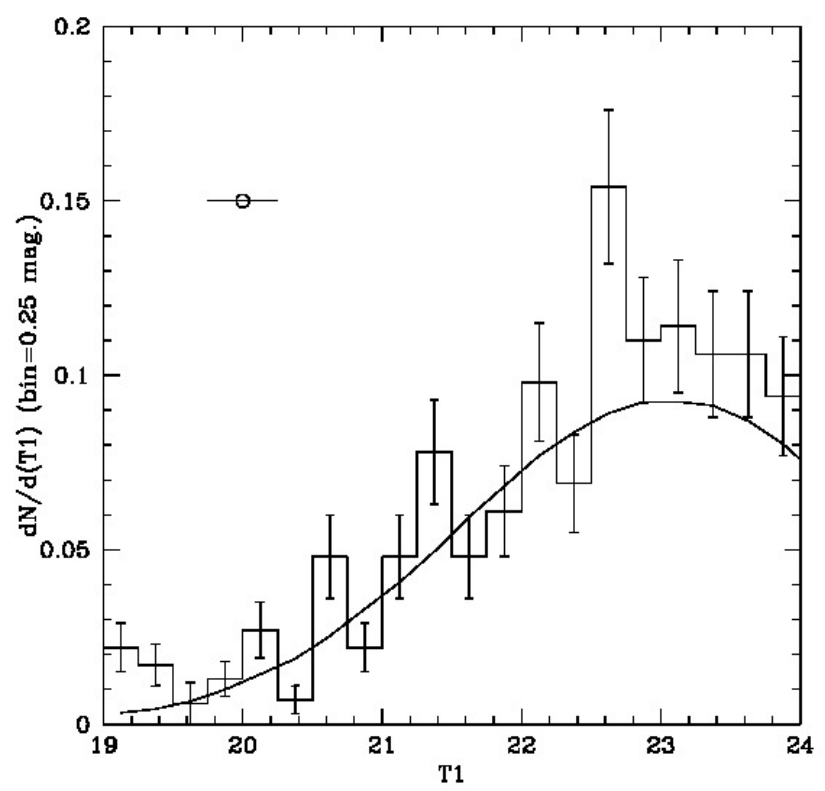

Figure A14. Similar to Fig. A6 but for $272 \mathrm{GC}$ candidates with $\left(C-T_{1}\right) o$ colours from 1.64 to 1.74 .

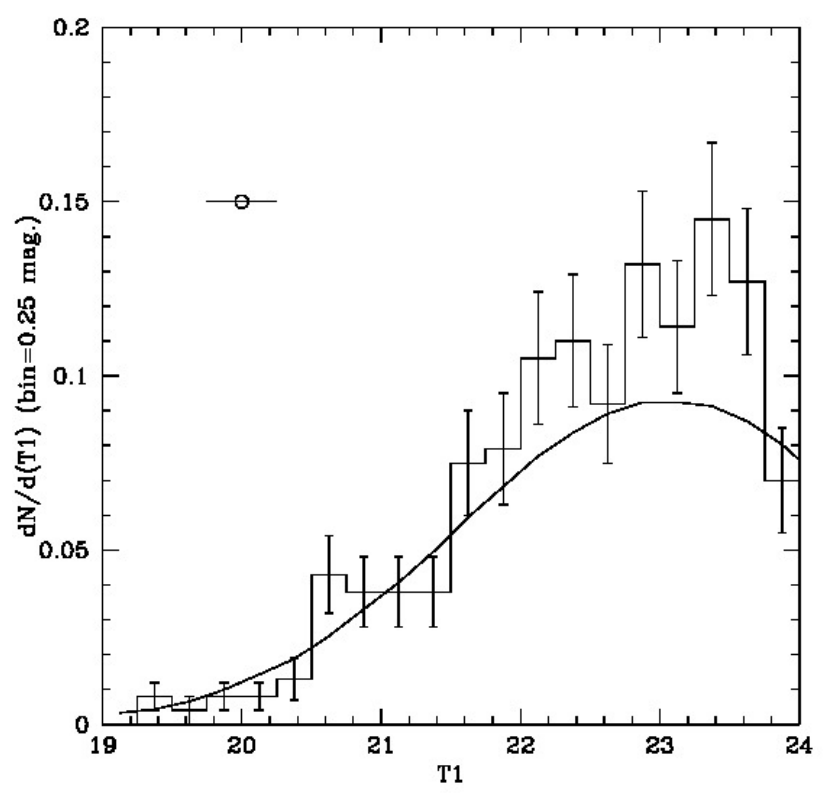

Figure A15. Similar to Fig. A6 but for $253 \mathrm{GC}$ candidates with $\left(C-T_{1}\right) o$ colours from 1.77 to 1.87 .

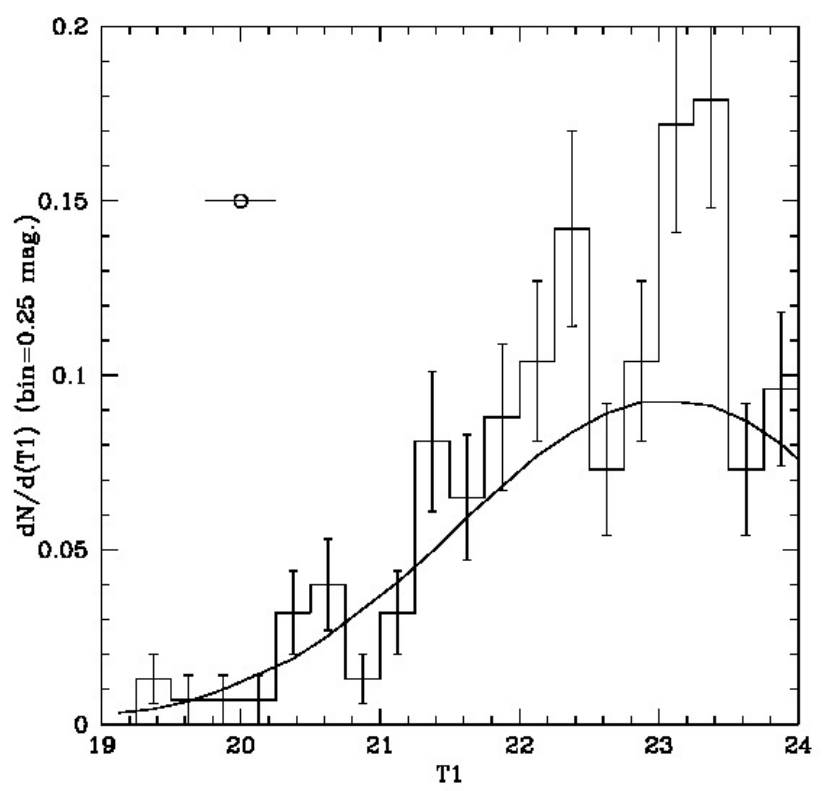

Figure A16. Similar to Fig. A6 but for 149 GC candidates with $\left(C-T_{1}\right) o$ colours from 1.88 to 1.98 . 